\title{
Simulation of Residual Stress and Elastic Energy Density in Thermal Barrier Coatings Using Fast Fourier Transforms
}

\author{
S.P. Donegan and A.D. Rollett
}

\author{
Sean P. Donegan* \\ BlueQuartz Software \\ 400 S Pioneer Blvd \\ Springboro, OH 45066 \\ Email: sean.donegan@bluequartz.net \\ Phone: 937-806-1175 \\ Anthony D. Rollett \\ Department of Materials Science and Engineering Carnegie Mellon University \\ 5000 Forbes Avenue \\ Wean Hall 3313 \\ Pittsburgh, PA 15213 \\ Email: rollett@andrew.cmu.edu \\ Phone: 412-268-3177
}

Fax: 412-268-3113

* : Corresponding Author

Keywords: thermal barrier coatings; residual stress; simulation; synthetic microstructures; fast Fourier transform

(C) 2015. This manuscript version is made available under the Elsevier user license

http://www.elsevier.com/open-access/userlicense/1.0/ 


\begin{abstract}
A numerical method for solving the thermoelastic problem in heterogeneous polycrystals based on fast Fourier transforms is applied to thermal barrier coating systems. Several high resolution microstructures are generated synthetically to approximate thermal barrier coatings, with control over the grain size, grain morphology, and texture. Interfaces between coating layer materials are further modified by applying a localized Potts model to introduce interface rumpling. The global results of residual stress and elastic energy density are compared across the various microstructure instantiations. The local variations in elastic energy density are correlated to the amount of interface rumpling. The simulation result are also compared to an analytical result for an idealized interface morphology. The implications of the behavior of the local variations in elastic energy density are discussed in the context of thermal barrier coating failure.
\end{abstract}

\title{
1 Introduction
}

Structural metallic components operating in gas turbine engines are often exposed to extreme levels of temperature $[1,2]$. In such systems, it is desirable to increase the operating temperature, thereby improving efficiency and performance $[3,4]$. However, the mechanical properties of the metallic component must remain constant at elevated temperatures to avoid the potential for failure [5]. In order to provide insulation and protection for the metallic component, it is common to apply a ceramic coat to the metallic surface in contact with the combustion gas. These thermal barrier coating (TBC) systems, along with sufficient internal cooling, are capable of reducing the surface temperature of the alloy up to $100{ }^{\circ} \mathrm{C}$ [6]. This allows operating temperatures above the melting point of the metallic component $\left(\sim 1300^{\circ} \mathrm{C}\right.$ for a nickel based superalloy) [6].

TBC systems consist of several layers, including the structural metallic substrate, a metallic bond coat (BC), a ceramic thermally grown oxide (TGO), and a ceramic top coat (TC). The entire TBC system is subject to extreme variations in temperature, resulting in the development of significant thermal residual stresses attributed to the thermal expansion mismatch between layers and the intrinsic anisotropy in each layer $[7,8]$. These residual stresses can lead to the development of defects at the interfaces between constituent TBC layers known as "rumpling" [9-11]. The rumpling is a result of the TGO attempting to relax the strain energy associated with its intrinsic residual stress. Due to the thermal expansion mismatch between the BC and TGO, the TGO will develop compressive stresses within the plane normal to the interface on cooling $[10,11]$. To accommodate these residual stresses, the TGO will attempt to lengthen itself by displacements out of the interface plane $[6,9]$. The resulting stresses will then redistribute around the rumpling features $[9,10]$. The undulations may also continue to grow via a process known as "ratcheting" $[9,12]$. The growth of such interface features causes stress localization, eventually leading to cracking at the BC/TGO or TGO/TC interfaces. The source of the macroscale cracking is thus tied to a microstructurally local phenomenon.

Experimental assessment of TBC failure is essential for determining a limiting lifetime for components, but does little to reveal the local origins of failure [13-16]. Additionally, nondestructive measurements of residual stress are generally restricted to the macroscale [17,18]. Modeling provides an opportunity to investigate local residual stresses in TBC systems. In the present analysis, we utilize a spectral approach based on fast Fourier transforms (FFTs) to solve the constitutive equations related to thermal residual stress. This approach provides the possibility for $\mathcal{O}(n \log n)$ scaling, where $n$ is the number of nodes utilized in the simulation [19]. Comparable finite element methods (FEM) generally scale as $\mathcal{O}\left(n^{2}\right)$ and require a meshing procedure, inevitably leading to overall longer compute times. Since the present technique is image based, no meshing is needed, allowing for complex microstructures to be assessed.

\section{The Thermoelastic FFT Method}

In classical linear elasticity, stress and strain are related via Hooke's Law:

$$
\sigma_{i j}=C_{i j k l} \epsilon_{k l}
$$

where $\sigma_{i j}$ is the stress tensor and $C_{i j k l}$ is the elastic stiffness tensor. Einstein notation is used to simplify the present tensor manipulations, where repeated indices indicate summations. Thermal expansion and elasticity are coupled together by the concept of eigenstrains. An eigenstrain is a strain that develops without the 
application of an external stress. Such stress-free strains arise when a material undergoes a volume change, such as from thermal expansion [20,21]. By rearranging equation 1, the total strain can be written as follows:

$$
\epsilon_{i j}(\mathbf{x})=C_{i j k l}^{-1}(\mathbf{x}) \sigma_{k l}(\mathbf{x})+\epsilon_{i j}^{*}(\mathbf{x})
$$

where $\epsilon_{i j}(\mathbf{x})$ is the total strain, $C_{i j k l}^{-1}(\mathbf{x})$ is the elastic compliance tensor, $C_{i j k l}^{-1}(\mathbf{x}) \sigma_{k l}(\mathbf{x})=\epsilon_{i j}^{e}(\mathbf{x})$ is the elastic strain, and $\epsilon_{i j}^{*}(\mathbf{x})$ is the eigenstrain. Here, the quantities are written as functions of a position vector, $\mathbf{x}$. In the present context, the eigenstrain is a thermal strain that results from the action of a temperature change:

$$
\epsilon_{i j}^{*}(\mathbf{x})=\alpha_{i j}(\mathbf{x}) \Delta T
$$

where $\alpha(\mathbf{x})$ is the thermal expansion coefficient tensor defined over space, which determines the change in eigenstrain for a unit temperature increment. Note that it is assumed that the thermal expansion coefficient remains constant over $\Delta T$ [22]. Equation 2 may be inverted to obtain a representation of stress is:

$$
\sigma_{i j}(\mathbf{x})=C_{i j k l}(\mathbf{x})\left(\epsilon_{k l}(\mathbf{x})-\epsilon_{k l}^{*}(\mathbf{x})\right)
$$

The sources of the residual stresses in TBCs include the thermal expansion mismatch between component layers, the inherent thermal expansion anisotropy of any non-cubic phases, and the volume change associated with the growth of the TGO. The growth stresses of the TGO are, however, no more than an order of magnitude smaller than the thermal residual stresses [6, 9, 23-25]. However, the numerical approach used here only models the stresses that develop due to the eigenstrains that arise from equation 3.

A solution to the thermoelastic problem involves solving equations 2 and 4 with prescribed boundary conditions, namely stress equilibrium $[26]$

$$
\sigma_{i j, j}(\mathbf{x})=0
$$

along with compatibility. Comma notation is now used in indices to indicate partial differentiation. Equation 5 represents local static equilibrium absent any real body forces. Stress equilibrium is required for the volume to remain static, while compatibility ensures that when the volume is subjected to a displacement field, a unique strain field is obtained. Compatibility requires that the volume remains well connected, precluding the development of voids. Assuming compatibility is satisfied, the following equation can be used as a second boundary condition [26]:

$$
\epsilon_{i j}(\mathbf{x})=\frac{1}{2}\left(u_{i, j}(\mathbf{x})+u_{j, i}(\mathbf{x})\right)
$$

The derivation of the solution to the thermoelastic problem using FFTs is described in detail by Anglin $[22,27]$. Begin by defining a homogeneous reference medium with corresponding elastic stiffness coefficients $C_{i j k l}^{\circ}$, which are the point average of $C_{i j k l}(\mathbf{x})$ over the entire volume. The use of a homogeneous reference medium was first described by Moulinec and Suquet [28] for use in computing the elastic response of composites. An overall strain state, $E_{i j}$, is applied to this homogeneous reference, resulting in local perturbations in the stress field, $\tau_{i j}(\mathbf{x})$. This perturbation field, commonly referred to as a polarization field, is a function of the heterogeneous elastic properties and thermal strains in the volume [22]:

$$
\tau_{i j}(\mathbf{x})=\left(C_{i j k l}(\mathbf{x})-C_{i j k l}^{\circ}\right) \epsilon_{k l}(\mathbf{x})-C_{i j k l}(\mathbf{x}) \epsilon_{k l}^{*}(\mathbf{x})
$$

Modifying Hooke's law with incorporated eigenstrains to include the homogeneous reference medium leads to the following equation:

$$
\sigma_{i j}(\mathbf{x})=C_{i j k l}^{\circ} \epsilon_{k l}(\mathbf{x})+\tau_{i j}(\mathbf{x})
$$

Combining equation 8 with equations 5 and 6 yields the following:

$$
C_{i j k l}^{\circ} u_{k, l j}(\mathbf{x})+\tau_{i j, j}(\mathbf{x})=0
$$

where $u_{k}$ is the displacement vector along the $x_{k}$ direction. A Green's function approach is used to solve equation 9 [29]. Replacing the displacement vector with the periodic Green's function, $G_{k m}\left(\mathbf{x}-\mathbf{x}^{\prime}\right)$ yields:

$$
C_{i j k l}^{\circ} G_{k m, l j}\left(\mathbf{x}-\mathbf{x}^{\prime}\right)+\delta_{i m} \delta\left(\mathbf{x}-\mathbf{x}^{\prime}\right)=0
$$


where $G_{k m, l j}\left(\mathbf{x}-\mathbf{x}^{\prime}\right)$ represents the displacement in the $x_{k}$ direction, $\delta_{i m}$ is the Kronecker delta, and $\delta\left(\mathbf{x}-\mathbf{x}^{\prime}\right)$ is the Dirac delta function. A singularity in the periodic Green's function exists at $\mathbf{x}=\mathbf{x}^{\prime}$; therefore, to ensure a solution a this point, fluctuation fields are solved for as opposed to absolute fields, hence the use of the homogeneous reference medium. In order to calculate the strain fields, the fluctuation of the displacement field must be found by applying the periodic Green's function to the perturbation in the stress field:

$$
\tilde{u}_{k}(\mathbf{x})=\int_{V} G_{k i}\left(\mathbf{x}-\mathbf{x}^{\prime}\right) \tau_{i j, j}\left(\mathbf{x}^{\prime}\right) \mathrm{d} \mathbf{x}^{\prime}
$$

Thus, the periodic Green's function is convolved with the perturbations in the stress field. By applying compatibility to equation 11, the fluctuation of the displacement field is converted to the fluctuation of the strain field:

$$
\tilde{\epsilon}_{i j}(\mathbf{x})=E_{i j}+\operatorname{sym}\left(\int_{V} G_{i k, j l}\left(\mathbf{x}-\mathbf{x}^{\prime}\right) \tau_{k l}\left(\mathbf{x}^{\prime}\right) \mathrm{d} \mathbf{x}^{\prime}\right)
$$

where sym indicates the symmetric part of the second-order tensor result of the convolution integral. In frequency space, convolution integrals become simple tensor products. Taking the Fourier transform of equation 10 yields the following:

$$
C_{i j k l}^{\circ} \xi_{l} \xi_{j} \hat{G}_{k m}(\mathbf{k})=\delta_{i m}
$$

where " ' indicates the Fourier transform and $\xi_{l}$ and $\xi_{j}$ are the $l^{t h}$ and $j^{\text {th }}$ components of the wave vector, respectively. Variables in Fourier space are now written with respect to $\mathbf{k}$ to differentiate from real space position $\mathbf{x}$. Rearranging equation 13 to find an explicit representation of the periodic Green's function in frequency space results in the following equation:

$$
\hat{\Gamma}_{i j k l}^{\circ}(\mathbf{k})=-\left(\xi_{p} \xi_{q} C_{i p k q}^{o}\right)^{-1} \xi_{j} \xi_{l}
$$

where $\hat{\Gamma}_{i j k l}^{\circ}(\mathbf{k})$ has replaced $\hat{G}_{i k, j l}(\mathbf{k})$.

An augmented Lagrangian method is used to improve the convergence of the thermoelastic FFT algorithm. This Lagrangian approach was first proposed by Michel et al. for use in linear and non-linear elasticity in composites [30,31]. The augmented Lagrangian solves an additional problem for the non-linear response of the volume at each iteration. Additional stress and strain fields, represented as $\lambda(\mathbf{x})$ and $e(\mathbf{x})$, respectively, are defined. The non-linear response equation for each iteration is then:

$$
\frac{\delta w}{\delta e}\left(\mathbf{x}, e^{i}\right)+C^{\circ}: e^{i}(\mathbf{x})=C^{\circ}: \epsilon^{i}(\mathbf{x})+\lambda^{i-1}(\mathbf{x})
$$

where $w$ is the elastic energy density and $\frac{\delta w}{\delta e}$ is $\sigma(\mathbf{x})$. Here, ' : ' represents a double tensor contraction over indices. The non-linear stress field for each iteration is expressed as follows:

$$
\lambda^{i}(\mathbf{x})=\lambda^{i-1}(\mathbf{x})+C^{\circ}:\left(\epsilon^{i}(\mathbf{x})-e^{i}(\mathbf{x})\right)
$$

Both the stress $\left(\lambda^{i}(\mathbf{x})\right)$ and strain $\left(e^{i}(\mathbf{x})\right)$ fields associated with the augmented Lagrangian will converge to their corresponding thermoelastic FFT representations $\left(\sigma^{i}(\mathbf{x})\right.$ and $\epsilon^{i}(\mathbf{x})$, respectively) [27].

Iterations for the thermoelastic FFT algorithm begin by initializing the total applied strain field, $E$, the local stress field, $\lambda(\mathbf{x})$, and the total local strain field, $e(\mathbf{x})$ :

$$
\begin{aligned}
E^{0} & =\left\langle\epsilon^{*}(\mathbf{x})\right\rangle-C^{\circ^{-1}}: \Sigma \\
\lambda^{0}(\mathbf{x}) & =C^{\circ}:\left(E^{0}-\epsilon^{*}(\mathbf{x})\right) \\
e^{0}(\mathbf{x}) & =E^{0}
\end{aligned}
$$

Since the only stresses of present interest are those due to the anisotropy in thermal expansion of neighboring grains, the overall stress state, $\Sigma$, is considered to be zero. Given the initialized fields above, iteration $i$ proceeds as follows:

1. $\tau^{i}(\mathbf{x})=\lambda^{i-1}(\mathbf{x})-C^{\circ}: e^{i-1}(\mathbf{x})$

Solve for the stress perturbation field given the previous iteration's augmented fields. 
2. $\hat{\tau}^{i}(\mathbf{k})=\mathcal{F}\left(\tau^{i}(\mathbf{x})\right)$

Transform the stress perturbation field into frequency space.

3. $\epsilon^{i}(\mathbf{x})=E^{i-1}+\operatorname{sym}\left(\mathcal{F}^{-1}\left(\hat{\Gamma}^{\circ}: \hat{\tau}^{i}(\mathbf{k})\right)\right)$

Solve for the total strain field in real space by computing the tensor multiplication of the frequency space Green's function and stress perturbation field and then applying an inverse Fourier transform.

4. $\sigma^{i}(\mathbf{x})+C^{\circ}:\left(C^{-1}(\mathbf{x}): \sigma^{i}(\mathbf{x})+\epsilon^{*}(\mathbf{x})\right)=\lambda^{i-1}(\mathbf{x})+C^{\circ}: \epsilon^{i}(\mathbf{x})$

$\sigma^{i}(\mathbf{x})=\left(I-C^{\circ}: C^{-1}(\mathbf{x})\right)\left[\lambda^{i-1}(\mathbf{x})+C^{\circ}:\left(\epsilon^{i}(\mathbf{x})-\epsilon^{*}(\mathbf{x})\right)\right]$

Solve for the total stress field in real space using the real space strain field and the augmented fields.

5. $e^{i}(\mathbf{x})=C^{-1}(\mathbf{x}): \sigma^{i}(\mathbf{x})+\epsilon^{*}(\mathbf{x})$

Solve for the current iteration's augmented strain field.

6. $\lambda^{i}(\mathbf{x})=\lambda^{i-1}(\mathbf{x})+C^{\circ}:\left(\epsilon^{i}(\mathbf{x})-e^{i}(\mathbf{x})\right)$

Solve for the current iteration's augmented stress field.

7. $E^{i}=\left\langle\epsilon^{i}(\mathbf{x})\right\rangle+C^{\circ^{-1}}:\left(\Sigma-\left\langle\sigma^{i}(\mathbf{x})\right\rangle\right)$

Recompute the overall strain state.

Iterations continue for either a preset amount or until a suitably small error is achieved. Errors in the stress and strain field errors are computed as follows:

$$
\begin{gathered}
\operatorname{err}\left[\lambda^{i}(\mathbf{x})\right]=\frac{\left\langle\left\|C^{\circ}:\left(\epsilon^{i}(\mathbf{x})-e^{i}(\mathbf{x})\right)\right\|\right\rangle}{\left\|\left\langle\sigma^{i}(\mathbf{x})\right\rangle\right\|} \\
\operatorname{err}\left[e^{i}(\mathbf{x})\right]=\frac{\left\langle\left\|\epsilon^{i}(\mathbf{x})-e^{i}(\mathbf{x})\right\|\right\rangle}{E}
\end{gathered}
$$

where $\left\|\sigma_{i}\right\|=\sqrt{\sigma_{i} \sigma_{i}}$ and $\langle$.$\rangle is the average for all \mathbf{x}$, with the same calculations for strain.

\section{Synthetic Structure Generation}

The thermoelastic FFT is image based, requiring a domain discretized by a regular grid, termed a Fourier grid. In the present work, a rectilinear grid is used for simplicity. Figure 1 shows an underlying 2D microstructure image and its discretization. 


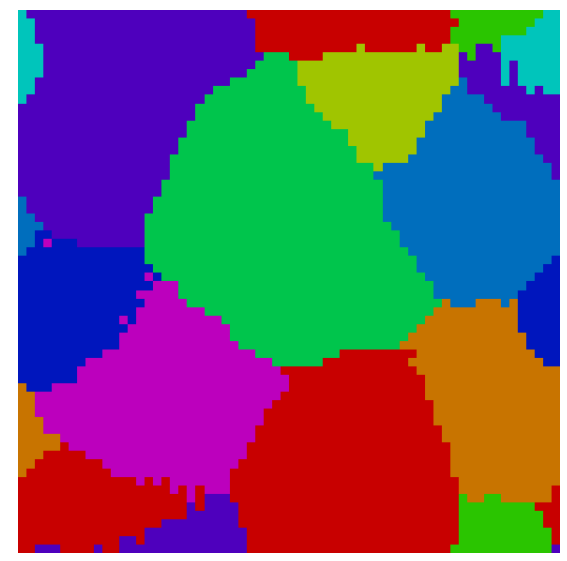

(a) Underlying sample microstructure.

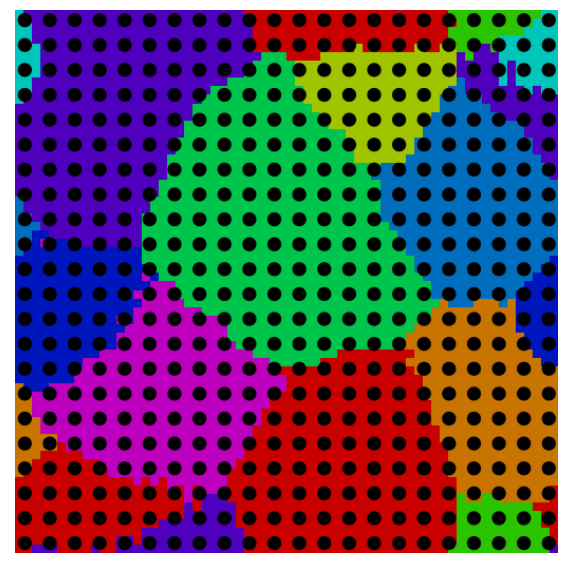

(b) Overlay of rectilinear grid.

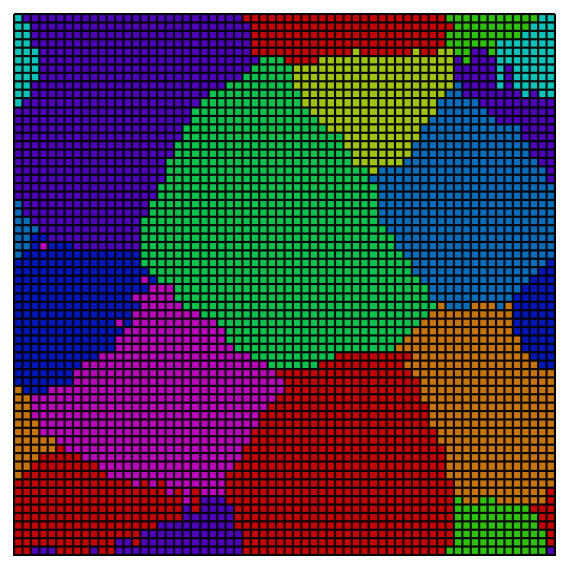

(c) Center-weighted pixelization.

Figure 1: A sample discretized 2D microstructure suitable for use as input to the thermoelastic FFT code.

The microstructure image may be obtained from experimental procedures or generated synthetically. Any experimentally measured microstructures must be obtained using a form of orientation imaging, such as EBSD, since the thermoelastic FFT method requires orientation information. The thickness of TBC systems makes 3D experimental characterization impractical. Additionally, a variety of interface morphologies are desirable, in order to assess the effect of local microstructural features. These issues are overcome by using synthetic microstructures. The digital microstructures presented here were generated using the DREAM.3D application [32]. To construct a synthetic microstructure, a set of descriptive statistics are required. These statistics include the grain size distribution, which includes the grain morphology, as defined by the ratios of the semiaxes of an ellipsoid, and the orientation distribution. Control over the grain morphology allows for both equiaxed and columnar grains to be created. Additionally, the texture can be varied by altering the orientation distribution function (ODF) and the axis orientation distribution function (axis ODF). Based on the input microstructure statistics, DREAM.3D packs pseudograins of a predetermined shape class and proceeds to iteratively grow them via a cellular automaton. Synthetic structures can be forced to be periodic (i.e., for a field $\mathbf{u}$ at time $t, \mathbf{u}(\mathbf{x}+\hat{i} L, t)=\mathbf{u}(\mathbf{x}, t)$ and $\mathbf{u}(\mathbf{y}+\hat{j} L, t)=\mathbf{u}(\mathbf{y}, t)$, where $L$ is the size of the domain). Since the thermoelastic FFT requires periodic boundaries, structures created within DREAM.3D are always forced to have periodic grain structures.

Although DREAM.3D supports the creation of multiphase microstructures, it does not support the explicit creation of layered structures, such as those seen in TBCs. To circumvent this, each layer of the composite TBC is generated separately. The resulting layers are then "stitched" together along the $z$-axis to form a continuous microstructure. The procedure requires that all layers share the same $x$ and $y$ dimensions. The stitching process is shown schematically in Figure 2. 


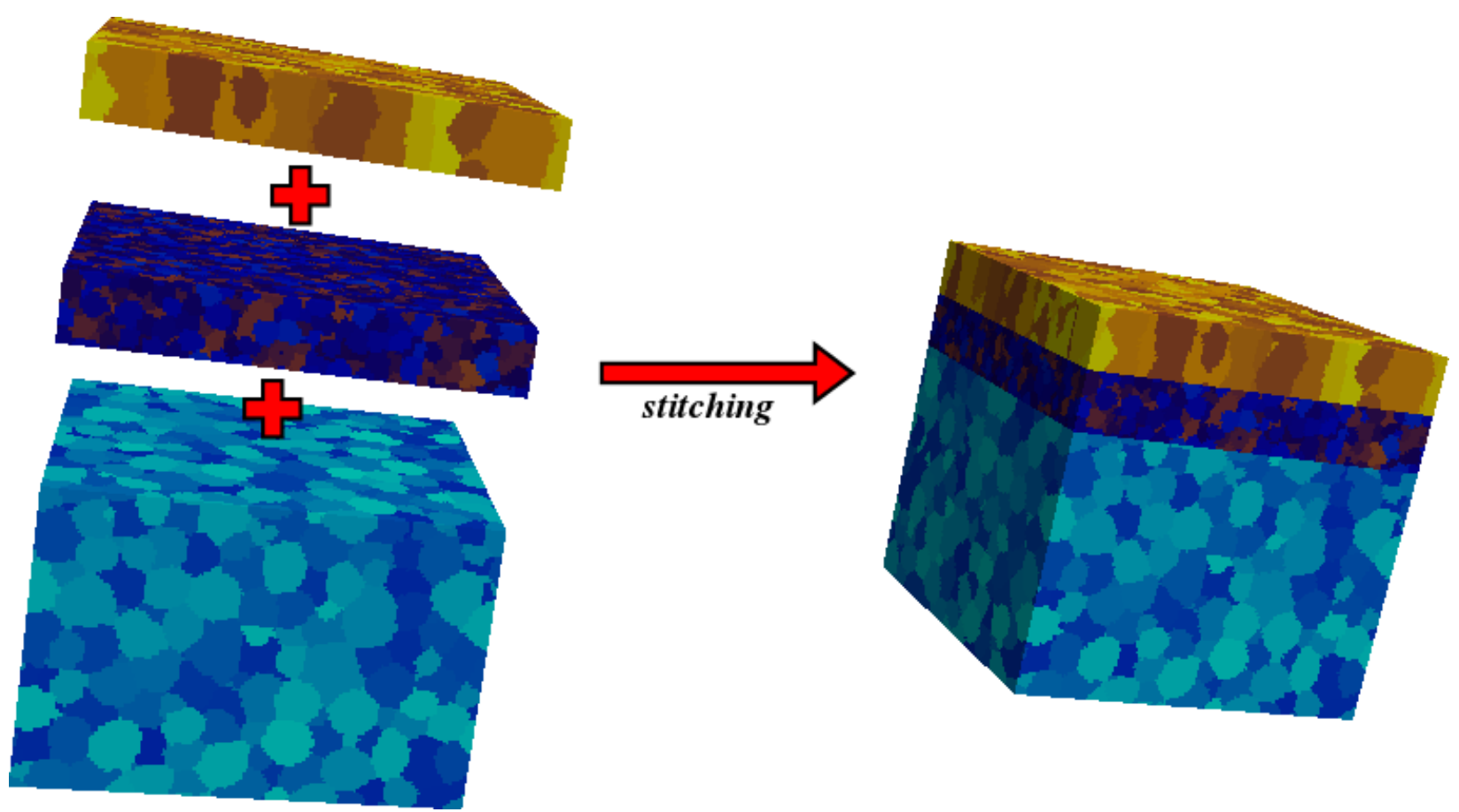

Figure 2: Stitching process used to create layered thermal barrier coating microstructures.

The interfaces created with the stitching process are inherently flat. To approximate rumpled interfaces, a localized Potts grain growth model is used to allow for grain relaxation near the layer interfaces.

The instantiation of the Potts model used is purely stochastic. The algorithm begins by selecting a random voxel within the digitized microstructure $\left(n_{1}\right)$. After choosing a voxel and determining its grain identification (or "spin"), the nearest neighbor voxels and their spins are identified. A random spin is chosen from this neighbor set $\left(n_{2}\right)$. To compute the energy change for a possible spin flip, the algorithm iterates over the set of nearest neighbors and compares each neighbor spin $\left(n_{3}\right)$ to the original spin $\left(n_{1}\right)$ and the randomly chosen neighbor spin $\left(n_{2}\right)$ [33]. The comparison between $n_{1}$ and $n_{2}$ produces an energy value of $E_{1}$ whereas the comparison between $n_{2}$ and $n_{3}$ produces an energy value of $E_{2}$. If spin values match, then an energy value of zero is assigned, whereas if spins values differ, an energy value of one is assigned [33]. The local energy change, $\mathrm{d} E$, is then $E_{2}-E_{1}$. The total energy change, $\mathrm{d} E_{t o t}$, is the sum of $\mathrm{d} E$ for all nearest neighbors. If $\mathrm{d} E_{t o t}$ is less than 0 , then a spin flip occurs, and $n_{1}$ becomes $n_{2}$. If, however, $\mathrm{d} E_{t o t}$ is greater than 0 , an additional comparison is made. A random value on the interval $[0,1]$ is chosen and compared to the Boltzmann factor, $e^{\frac{d E_{t o t}}{k T}}$. If the random value is less than the Boltzmann factor, a spin flip also occurs. $k T$ here represents a lattice temperature that controls interface roughness by allowing fluctuations in position [33]. If neither of these conditionals is met, no spin flip occurs.

A total of eight high resolution synthetic microstructures were generated for this study. Four of the structures utilize a columnar TC, associated with manufacturing via electron beam physical vapor deposition (EB-PVD). The remaining four microstructures exhibit a splat TC, associated with manufacturing via air plasma spraying (APS). Additionally, the texture within the TGO was varied as either random or fiber. Finally, interface rumpling was introduced in half the microstructures using the above localized Potts model technique. Grain maps for the eight microstructures are shown in Figure 3. 


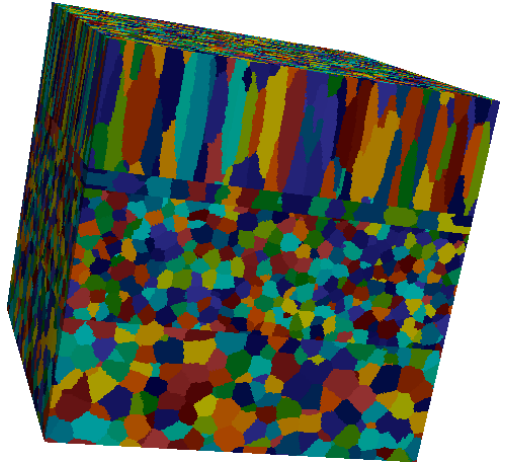

(a) Columnar TC, randomly textured TGO, and flat interfaces.

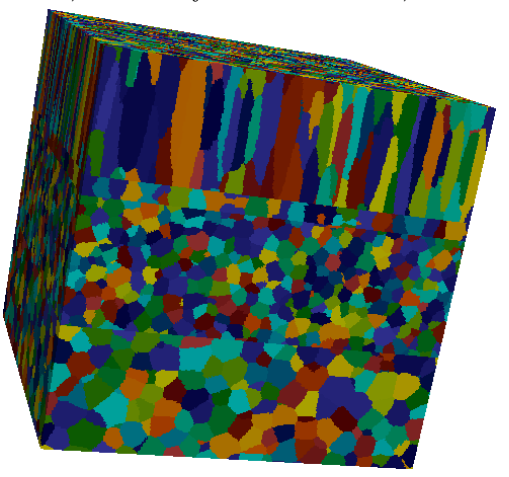

(c) Columnar TC, fiber textured TGO, and flat interfaces.

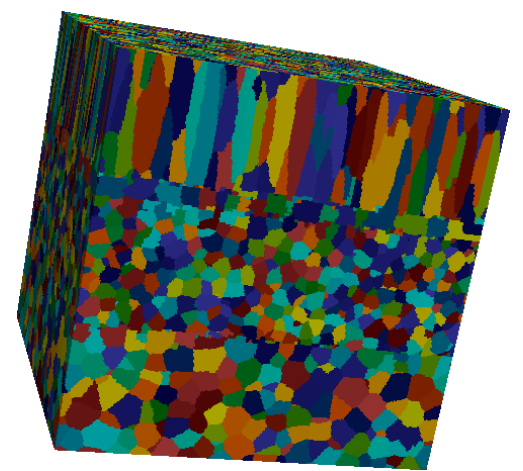

(b) Columnar TC, randomly textured TGO, and rumpled interfaces.

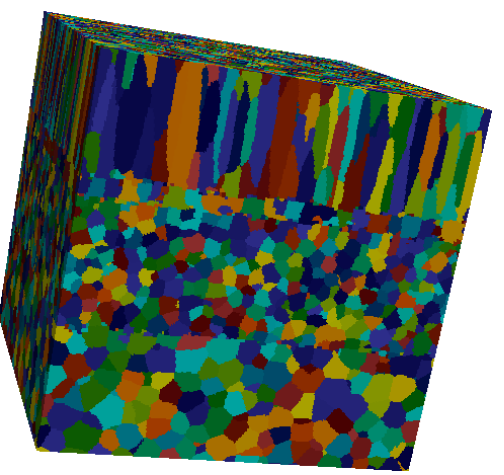

(d) Columnar TC, fiber textured TGO, and rumpled interfaces. 


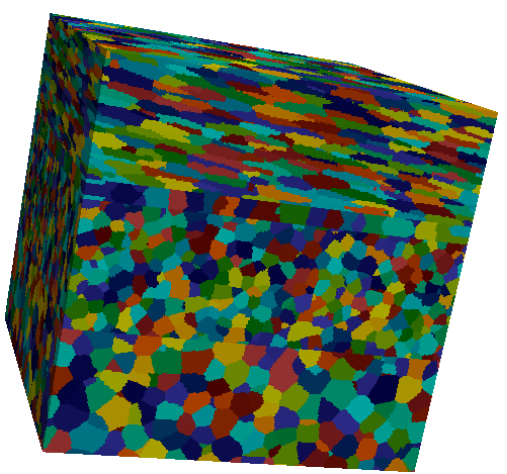

(e) Splat TC, randomly textured TGO, and flat interfaces.

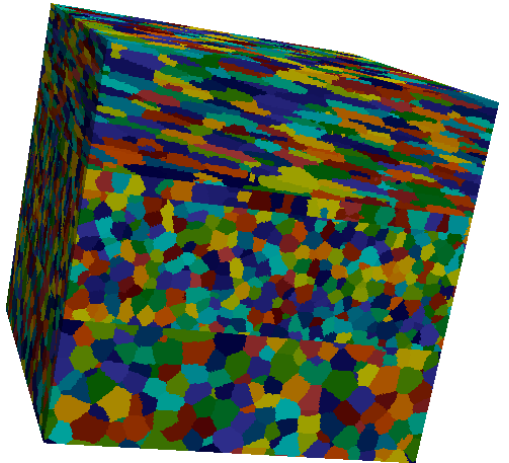

(g) Splat TC, fiber textured TGO, and flat interfaces.

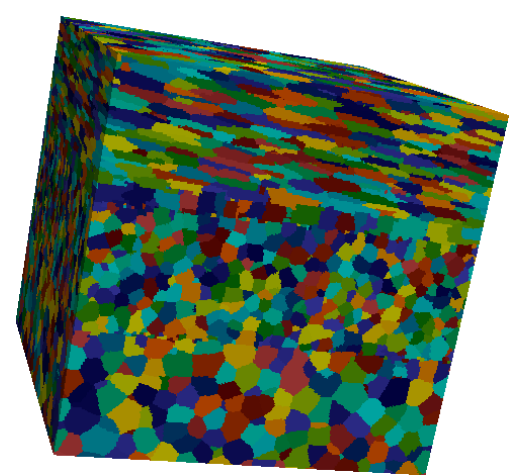

(f) Splat TC, randomly textured TGO, and rumpled interfaces.

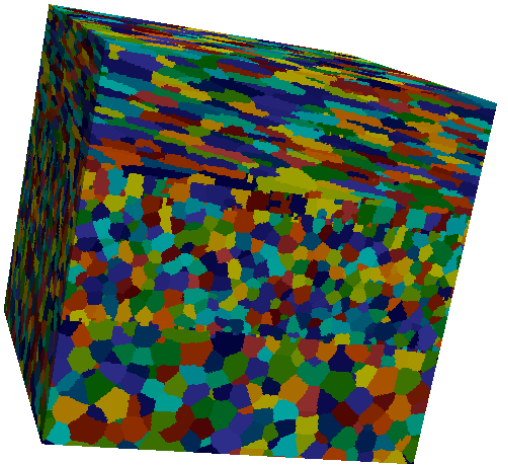

(h) Splat TC, fiber textured TGO, and rumpled interfaces.

Figure 3: Grain maps of the eight high resolution microstructures used in the full TBC analysis.

The above microstructures are all instantiated on $315^{3}$ Fourier grids. The substrate, BC, and TC are each 100 voxels thick, while the TGO is 15 voxels thick, for all microstructures. This ratio roughly corresponds to the actual ratio of thicknesses in real TBC microstructures $[6,9]$. The substrate and BC are each composed of nominally equiaxed grains with random textures. The TGO in all cases is composed of columnar grains. The morphology of the TC grains depends on the deposition technique. For the columnar TCs, a strong fiber texture was enforced. Figure 4 shows example inverse pole figure (IPF) maps of the constituent layers of a full synthetic TBC microstructure, with respect to the $z$ direction.

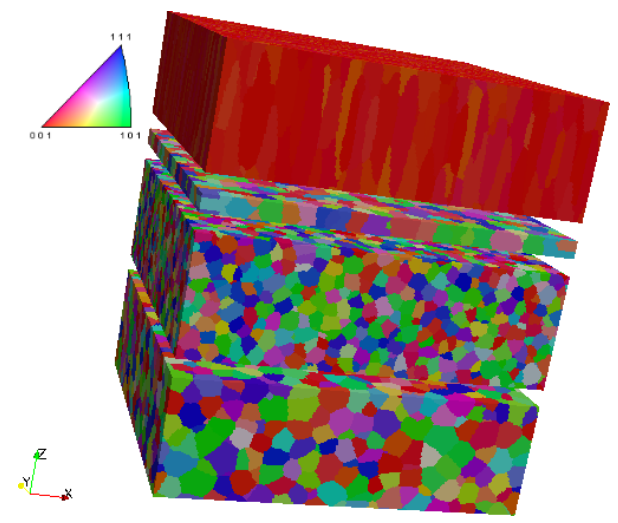

(a) Microstructure with a randomly textured TGO.

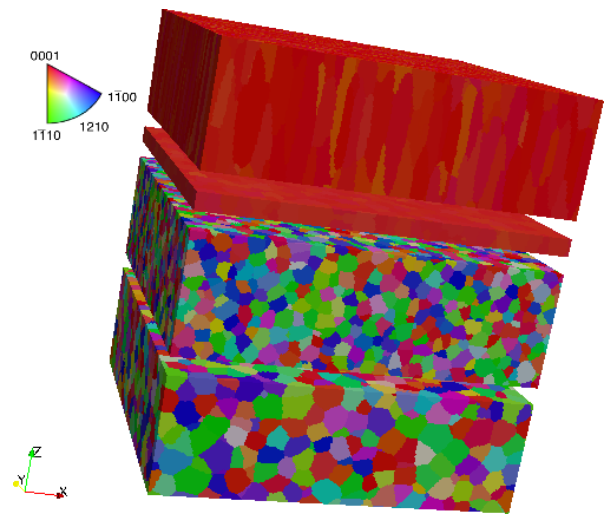

(b) Microstructure with a fiber textured TGO.

Figure 4: Example IPF maps of the constituent synthetic TBC layers. Standard stereographic triangles are displayed for cubic systems (left) and trigonal systems (right).

The microstructures contained periodic grains to conform to the thermoelastic FFT method. To simulate 
a free surface, a five voxel thick buffer layer was applied to the top of the TC. The buffer layer serves to circumvent the periodic boundary conditions by introducing a region with infinite elastic compliance, which is achieved by enforcing zero stress [34]. The interface rumpling was induced via the localized Potts model approach described above. The layers \pm 5 voxels away from the initially flat interface were allowed to evolve. A clearer view of the interface rumpling is shown in Figure 5.

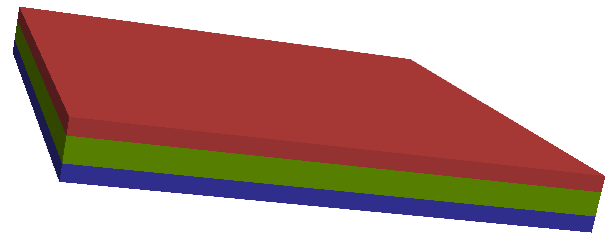

(a) Interfaces before localized Potts model.

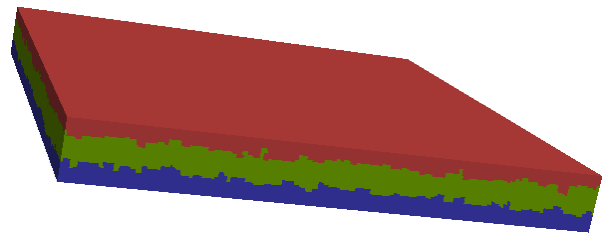

(b) Interfaces after localized Potts model.

Figure 5: Example phase maps near the TGO displaying the effect of interface rumpling.

The images in Figure 5 are example subsets of the images in Figure 3 for regions near the TGO, colored by phase type. The rumpling is clearly visible, indicating that the localized Potts model has the capability to induce significant morphological variation in the $\mathrm{BC} / \mathrm{TGO}$ and $\mathrm{TGO} / \mathrm{TC}$ interfaces.

Industry standard materials were chosen for the present analysis, shown in Table 1.

\begin{tabular}{l|c|c|c} 
TBC Component & Material & Crystal Structure & $\alpha^{\text {aniso }}$ \\
\hline \hline Top Coat & YSZ & cubic & 1 \\
\hline \multirow{2}{*}{ Bond Coat } & NiCoCrAlY & cubic & 1 \\
& cubic & 1 \\
\hline TGO & $\alpha-\mathrm{Al}_{2} \mathrm{O}_{3}$ & trigonal & 1.11 \\
\hline Substrate & $\mathrm{IN718}$ & cubic & 1
\end{tabular}

Table 1: Materials and their structural properties used for the full TBC analysis.

The metric $\alpha^{\text {aniso }}$ is the ratio of the $c$-axis thermal expansion coefficient $\left(\alpha^{c}\right)$ and the $a$-axis thermal expansion coefficient $\left(\alpha^{a}\right)$, which provides a measure of the amount of thermal anisotropy of the material. In addition to the thermal expansion coefficients of each material, the complete elastic stiffness tensor is also required. Table 2 lists the elastic stiffness coefficients for those materials listed in Table 1.

\begin{tabular}{l|c|c|c|c|c|c|c} 
TBC Component & Material & $C_{11}$ & $C_{12}$ & $C_{13}$ & $C_{14}$ & $C_{33}$ & $C_{44}$ \\
\hline \hline Top Coat & YSZ & 204 & 87 & - & - & - & 158 \\
\hline \multirow{2}{*}{ Bond Coat } & NiCoCrAlY & 269.2 & 115.4 & - & - & - & 76.9 \\
& (Ni,Pt)Al & 199 & 137 & - & - & - & 116 \\
\hline TGO & $\alpha-\mathrm{Al}_{2} \mathrm{O}_{3}$ & 497 & 163 & 116 & 22 & 501 & 147 \\
\hline Substrate & $\mathrm{IN718}$ & 339.5 & 173.3 & - & - & - & 21.4
\end{tabular}

Table 2: Elastic stiffness coefficients of the materials used for the full TBC analysis. Units are GPa.

Since literature data for the elastic stiffness tensor of NiCoCrAlY is sparse, the full set of coefficients were 
calculated from the isotropic elastic constants from the following equations:

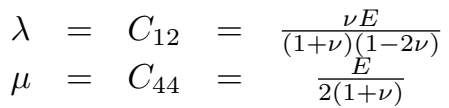

where $\lambda$ is the Lamé constant, $\mu$ is the shear modulus, $E$ is the Young's modulus, and $\nu$ is Poisson's ratio. Average $E$ and $\nu$ values for two phase NiCoCrAlY were utilized [9]. The component $C_{11}$ of the elastic stiffness tensor can be determined from the following relation:

$$
C_{44}=\frac{1}{2}\left(C_{11}-C_{12}\right)
$$

\section{Results}

The thermoelastic FFT was applied to the microstructures shown in Figure 3 with an enforced $\Delta \mathrm{T}=1000$ K. The algorithm described in Section 2 involves a macroscopic strain state, $E$. Since the present use case is concerned primarily with the stresses that develop internal to the microstructure, the overall stress state, $\Sigma$, is considered to be zero. This assumption reduces the initialization equations 17 for the FFT algorithm:

$$
\begin{aligned}
E^{0} & =\left\langle\epsilon^{*}(\mathbf{x})\right\rangle \\
\lambda^{0}(\mathbf{x}) & =C^{\circ}:\left(E^{0}-\epsilon^{*}(\mathbf{x})\right) \\
e^{0}(\mathbf{x}) & =E^{0} \\
C^{\circ} & =\langle C(\mathbf{x})\rangle
\end{aligned}
$$

where $\langle$.$\rangle denotes averaging over all \mathbf{x}$. Since two BC materials were chosen for analysis, a total of sixteen simulations were conducted. The results are presented with reference to the morphology of the TC.

\subsection{Columnar Top Coats}

The maximum principal stress, defined as the largest eigenvalue of the resulting stress tensor, was calculated at each Fourier point. Figure 6 shows the maximum principal stress maps for the eight columnar TC simulations.

The color maps in Figure 6 are not drawn to the same scale to highlight individual features of each microstructure. This visually highlights the local variations of the individual microstructures, as opposed to making direct comparisons between the different microstructures. Figures 6 (a-d) display zero maximum principal stress within the bulk of the $\mathrm{BC}$, with fluctuations away from zero near the substrate/BC and $\mathrm{BC} / \mathrm{TGO}$ interfaces. These features are not unexpected, since these simulations were run using NiCoCrAlY as the BC material. Since isotropic elastic constants and thermal expansion coefficients were used for NiCoCrAlY, no residual stress is expected in the BC bulk. Fluctuations away from zero are therefore due to the thermal expansion mismatch between the $\mathrm{BC}$ and its neighboring layers (i.e., the substrate and the TGO).

The highest values of maximum principal stress are observed within the TGO layer. Additionally, for the microstructures containing rumpled interfaces, peaks in maximum principal stress appear to occur near ridges and troughs. In general, higher values of maximum principal stress are observed for microstructures with fiber textured TGOs and rumpled interfaces. Table 3 lists the highest maximum principal stress observed for each of the simulations shown in Figure 6. 


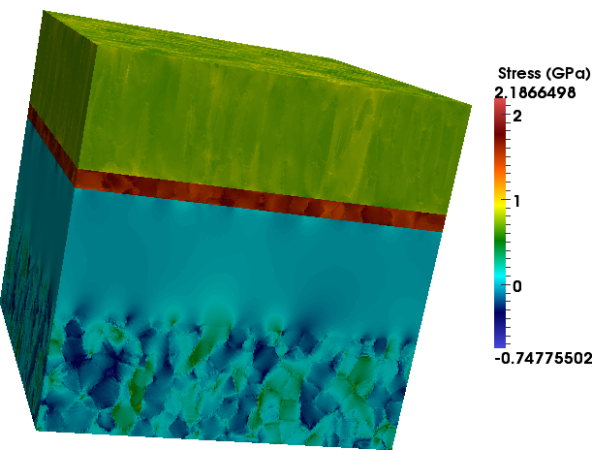

(a) NiCoCrAlY BC, randomly textured TGO, and flat inter- (b) faces.

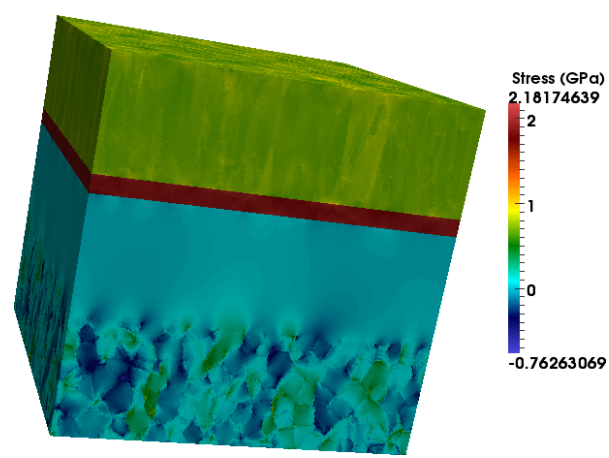

(c) NiCoCrAlY BC, fiber textured TGO, and flat interfaces.

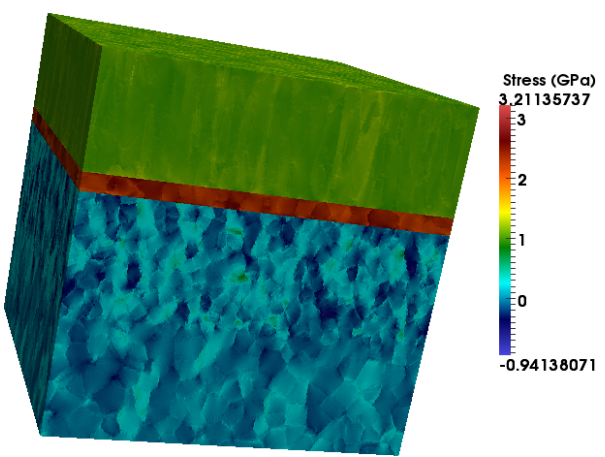

(e) $(\mathrm{Ni}, \mathrm{PT}) \mathrm{Al} \mathrm{BC}$, randomly textured TGO, and flat interfaces.

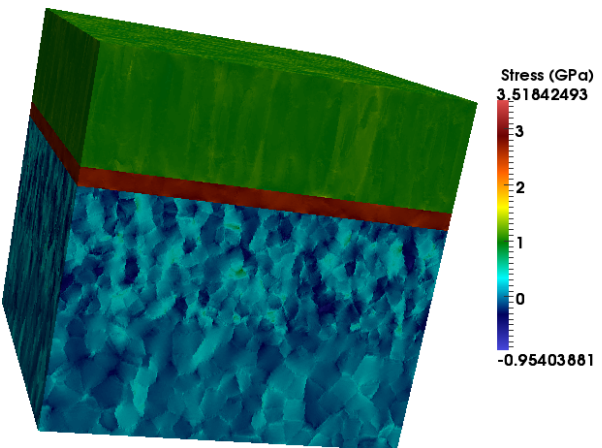

(g) (Ni,PT)Al BC, fiber textured TGO, and flat interfaces.

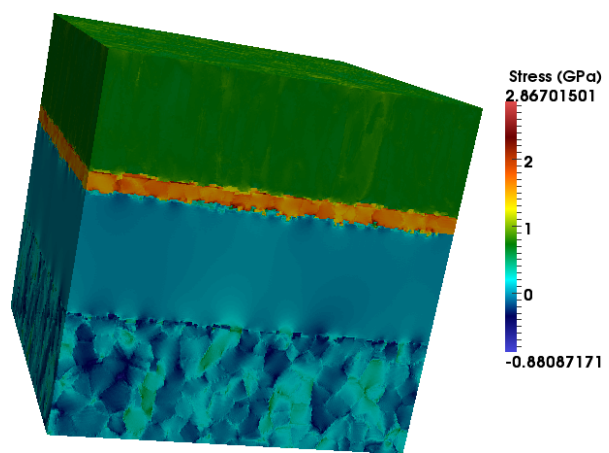

NiCoCrAlY BC, randomly textured TGO, and rumpled interfaces.

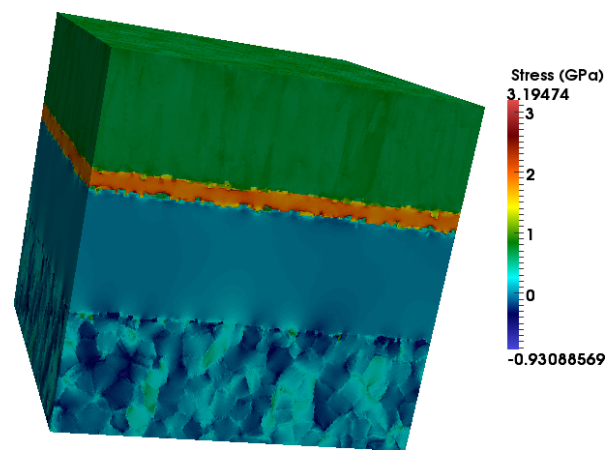

(d) NiCoCrAlY BC, fiber textured TGO, and rumpled interfaces.

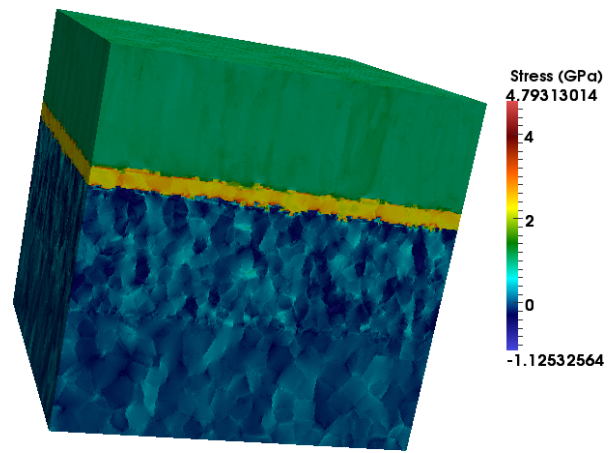

(f) $(\mathrm{Ni}, \mathrm{PT}) \mathrm{Al} \mathrm{BC}$, randomly textured $\mathrm{TGO}$, and rumpled interfaces.

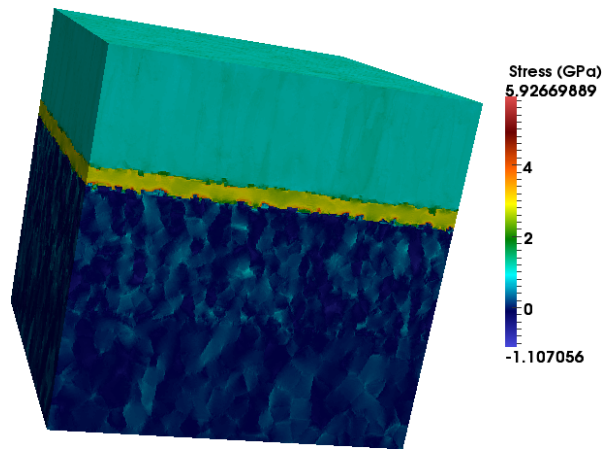

(h) $(\mathrm{Ni}, \mathrm{PT}) \mathrm{Al} \mathrm{BC}$, fiber textured TGO, and rumpled interfaces.

Figure 6: Maximum principal stress maps of the eight high resolution microstructures possessing columnar TCs. 


\begin{tabular}{l|c|c|c} 
Interface Type & BC Material & TGO Texture & Maximum Stress (GPa) \\
\hline \hline \multirow{4}{*}{ flat } & NiCoCrAlY & random & 2.187 \\
& NiCoCrAlY & fiber & 2.812 \\
& (Ni,Pt)Al & random & 3.211 \\
& (Ni,Pt)Al & fiber & 3.518 \\
\hline \multirow{4}{*}{ rumpled } & NiCoCrAly & random & 2.867 \\
& NiCoCrAlY & fiber & 3.195 \\
& (Ni,Pt)Al & random & 4.793 \\
& (Ni,Pt)Al & fiber & 5.927
\end{tabular}

Table 3: Highest maximum principal stress for each of the simulations shown in Figure 6.

The values listed in Table 3 indicate that microstructures with rumpled interfaces exhibit higher peak maximum principal stress than microstructures with flat interfaces. Similarly, microstructures with fiber textured TGOs display higher peak maximum principal stress than microstructures with randomly textured TGOs. Additionally, the (Ni,Pt)Al BCs display higher peak maximum principal stresses when compared to NiCoCrAlY BCs. This trend may simply be due to the isotropic properties assigned to the NiCoCrAlY system.

In addition to the maximum principal stress, the elastic energy density (EED) for each of the above eight simulations was calculated at each Fourier point. Figure 7 shows the EED maps for the eight columnar TC simulations.
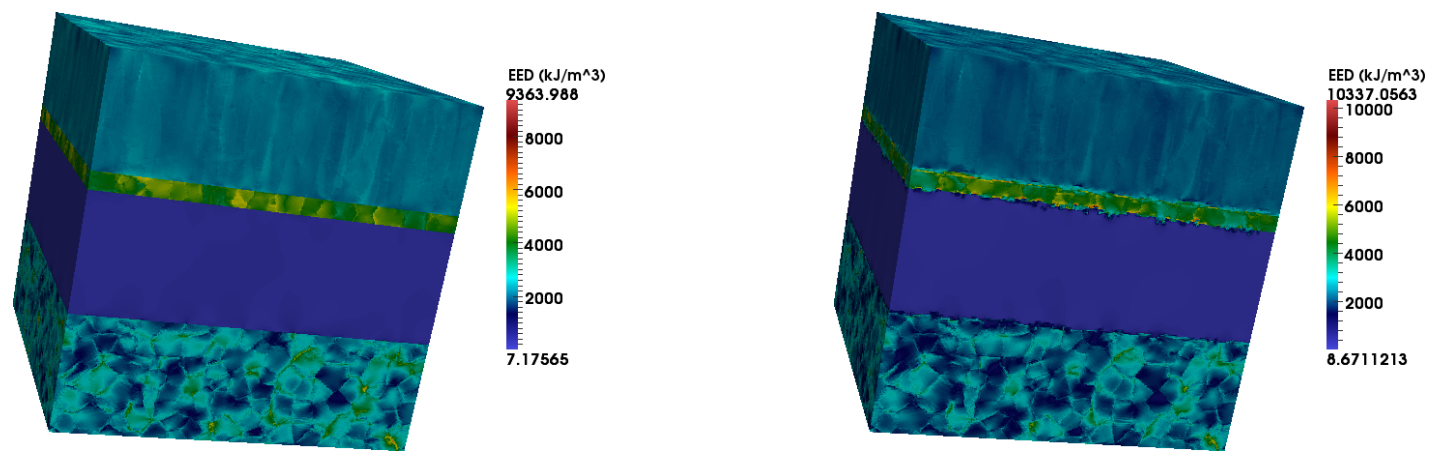

(a) NiCoCrAlY BC, randomly textured TGO, and flat inter- (b) NiCoCrAlY BC, randomly textured TGO, and rumpled infaces. terfaces. 


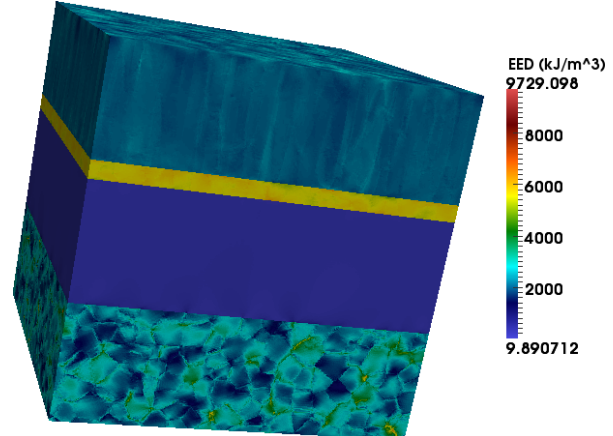

(c) NiCoCrAlY BC, fiber textured TGO, and flat interfaces.

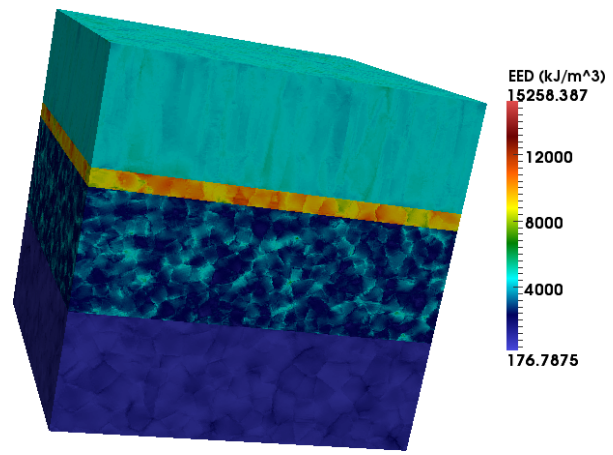

(e) $(\mathrm{Ni}, \mathrm{PT}) \mathrm{Al} \mathrm{BC}$, randomly textured TGO, and flat interfaces.

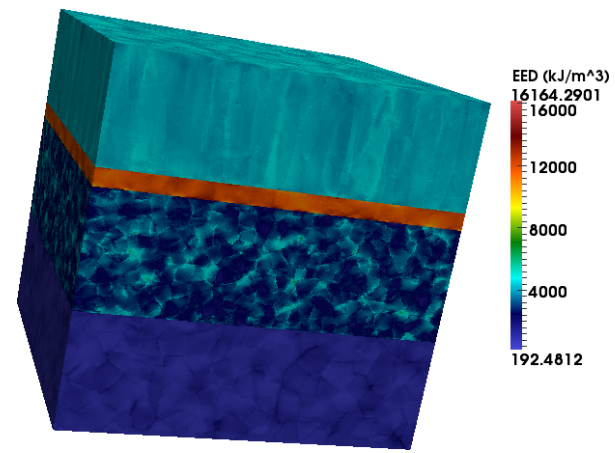

(g) $(\mathrm{Ni}, \mathrm{PT}) \mathrm{Al} \mathrm{BC}$, fiber textured TGO, and flat interfaces.

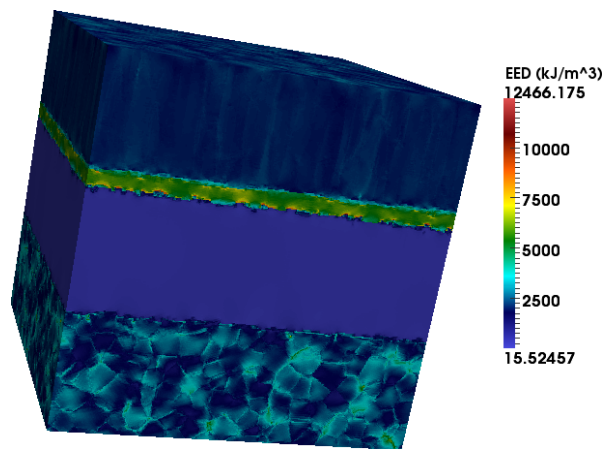

(d) NiCoCrAlY BC, fiber textured TGO, and rumpled interfaces.

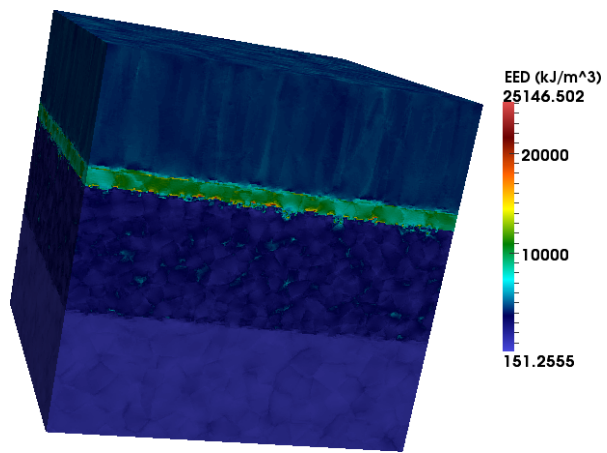

(f) $(\mathrm{Ni}, \mathrm{PT}) \mathrm{Al} \mathrm{BC}$, randomly textured TGO, and rumpled interfaces.

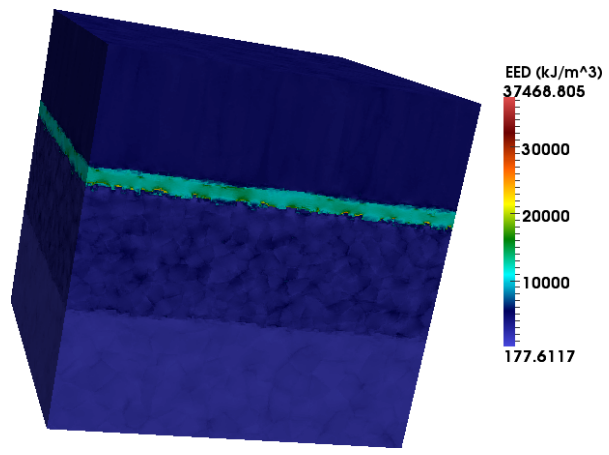

(h) $(\mathrm{Ni}, \mathrm{PT}) \mathrm{Al} \mathrm{BC}$, fiber textured TGO, and rumpled interfaces.

Figure 7: EED maps of the eight high resolution microstructures possessing columnar TCs.

Similar to Figure 6, the color maps in Figure 7 are not drawn to the same scale to highlight individual features of each microstructure. Figures 7 (a-d) display small amounts of EED within the bulk of the BC, again associated with the nearly zero level of residual stress in the $\mathrm{BC}$ due to the enforced isotropic properties of NiCoCrAlY.

The highest levels of stored strain energy are observed within the TGO, which agrees with the results from Figure 6. Peaks in EED appear near ridges and troughs for the microstructures with rumpled interfaces. The maximum EED follows the same trends observed for the maximum principal stress: higher values in the microstructures with fiber textured TGOs and rumpled interfaces. Table 4 lists the maximum EED for each of the simulations shown in Figure 7. 


\begin{tabular}{l|c|c|c} 
Interface Type & BC Material & TGO Texture & Maximum EED $\left(\frac{\mathrm{kJ}}{\mathrm{m}^{3}}\right)$ \\
\hline \hline \multirow{4}{*}{ flat } & NiCoCrAlY & random & 9364 \\
& NiCoCrAlY & fiber & 9729 \\
& (Ni,Pt)Al & random & 15258 \\
& (Ni,Pt)Al & fiber & 16164 \\
\hline \multirow{5}{*}{ rumpled } & NiCoCrAly & random & 10337 \\
& NiCoCrAlY & fiber & 12466 \\
& (Ni,Pt)Al & random & 25146 \\
& (Ni,Pt)Al & fiber & 37468
\end{tabular}

Table 4: Highest EED for each of the simulations shown in Figure 7.

The values listed in Table 4 indicate that EED follows the same trend as maximum principal stress: microstructures with rumpled interfaces or fiber textured TGOs exhibit higher maximum EED than microstructures with flat interfaces or randomly textured TGOs. Maximum EED is also significantly higher for the $(\mathrm{Ni}, \mathrm{Pt}) \mathrm{Al} \mathrm{BCs}$ when compared to the NiCoCrAlY BCs.

\subsection{Splat Top Coats}

The maximum principal stress maps for the eight splat TC simulations are shown in Figure 8. 


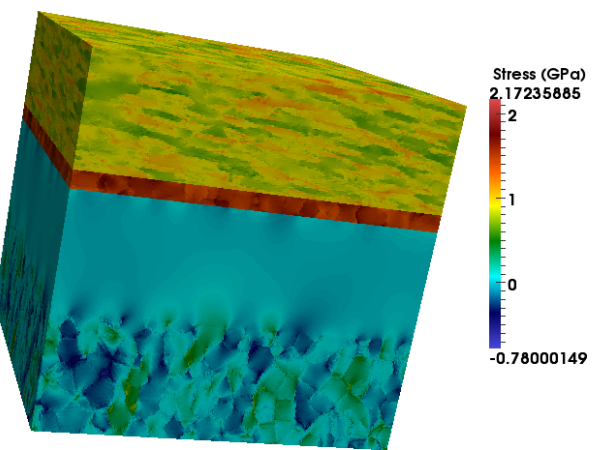

(a) NiCoCrAlY BC, randomly textured TGO, and flat inter- (b) faces.

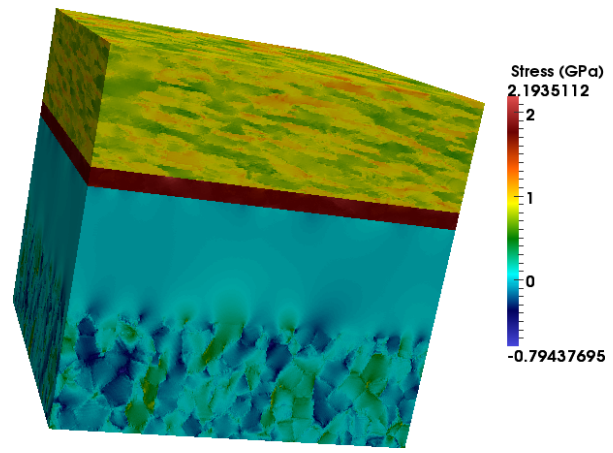

(c) NiCoCrAlY BC, fiber textured TGO, and flat interfaces

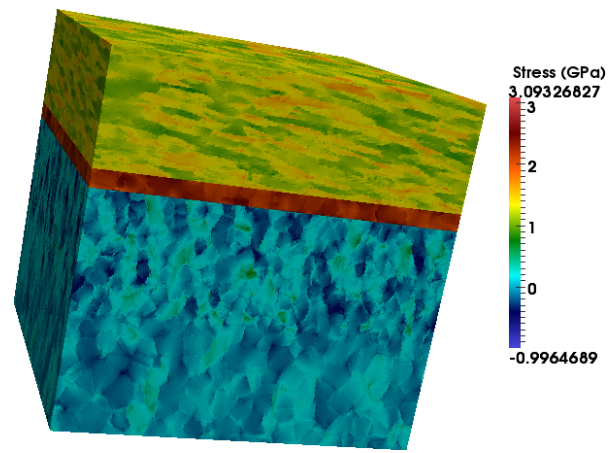

(e) $(\mathrm{Ni}, \mathrm{PT}) \mathrm{Al} \mathrm{BC}$, randomly textured TGO, and flat interfaces.

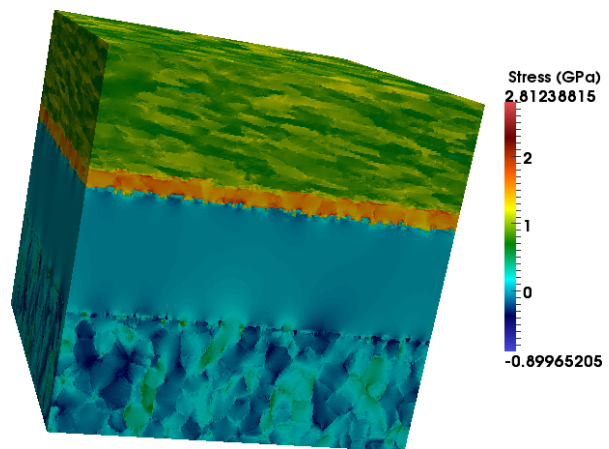

NiCoCrAlY BC, randomly textured TGO, and rumpled interfaces.

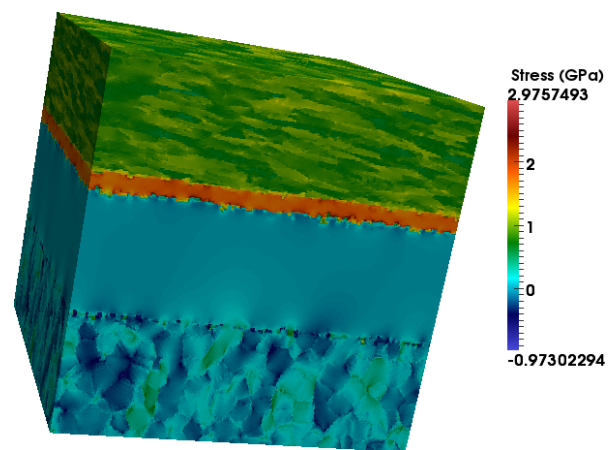

(d) NiCoCrAlY BC, fiber textured TGO, and rumpled interfaces.

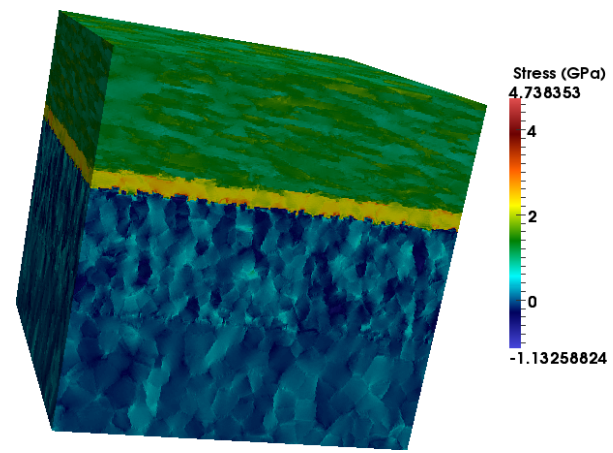

(f) $(\mathrm{Ni}, \mathrm{PT}) \mathrm{Al} \mathrm{BC}$, randomly textured TGO, and rumpled interfaces. 


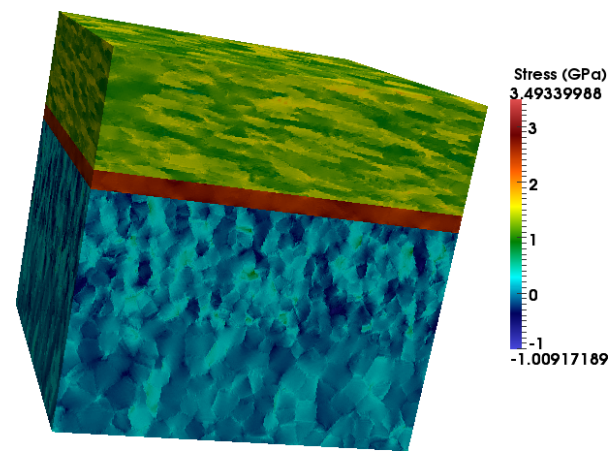

(g) $(\mathrm{Ni}, \mathrm{PT}) \mathrm{Al} \mathrm{BC}$, fiber textured TGO, and flat interfaces.

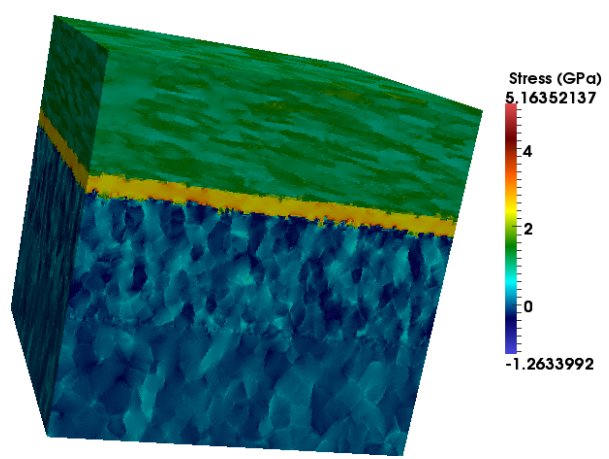

(h) $(\mathrm{Ni}, \mathrm{PT}) \mathrm{Al} \mathrm{BC}$, fiber textured TGO, and rumpled interfaces.

Figure 8: Maximum principal stress maps of the eight high resolution microstructures possessing splat TCs.

The color maps in Figure 8 are not drawn to the same scale to highlight individual features of each microstructure. As with the simulations involving columnar TCs, Figures 8 (a-d) display zero maximum principal stress within the bulk of the $\mathrm{BC}$, with fluctuations away from zero near the substrate/BC and BC/TGO interfaces. This feature is again explained by the isotropic properties used for NiCoCrAlY.

As with the columnar TCs, the highest values of maximum principal stress are observed within the TGO layer. The amount of residual stress within the TGO is roughly similar to that for the TGOs in Figure 6. Peaks in maximum principal stress are again observed near ridges and troughs for the microstructures with rumpled interfaces. The level of maximum principal stress in the splat TCs is similar to that of the columnar TCs, indicating that the morphology of the TC has little overall effect on the residual stress in that layer.

The peak values of maximum principal stress follow similar patterns to those in the microstructures with columnar TCs. Table 5 lists the highest maximum principal stress observed for each of the simulations shown in Figure 8.

\begin{tabular}{l|c|c|c} 
Interface Type & BC Material & TGO Texture & Maximum Stress (GPa) \\
\hline \hline \multirow{4}{*}{ flat } & NiCoCrAlY & random & 2.172 \\
& NiCoCrAlY & fiber & 2.193 \\
& (Ni,Pt)Al & random & 3.093 \\
& (Ni,Pt)Al & fiber & 3.493 \\
\hline \multirow{4}{*}{ rumpled } & NiCoCrAly & random & 2.812 \\
& NiCoCrAlY & fiber & 2.976 \\
& (Ni,Pt)Al & random & 4.738 \\
& (Ni,Pt)Al & fiber & 5.163
\end{tabular}

Table 5: Highest maximum principal stress for each of the simulations shown in Figure 8.

The values listed in Table 8 confirm the trends seen for columnar TCs. The peak maximum principal stress in the splat TC microstructures is generally smaller than that for the columnar TC microstructures. This feature may be related to the difference in grain morphologies at the TGO/TC interface. In columnar TCs, a strong fiber texture is typically found, such that the $c$-axes of the TC grains lie nearly parallel to the $c$-axes of the TGO grains. The splat TCs do not have this enforced texture, and thus any given orientation may exist within the TC. For the columnar TCs, the TGO grains and TC grains will push directly against each other as they thermally expand, resulting in higher peak residual stresses. This behavior is not possible in the splat TCs due to their grain morphology.

The EED for each of the above eight simulations was also calculated at each Fourier point. Figure 9 shows the EED maps for the eight splat TC simulations. 


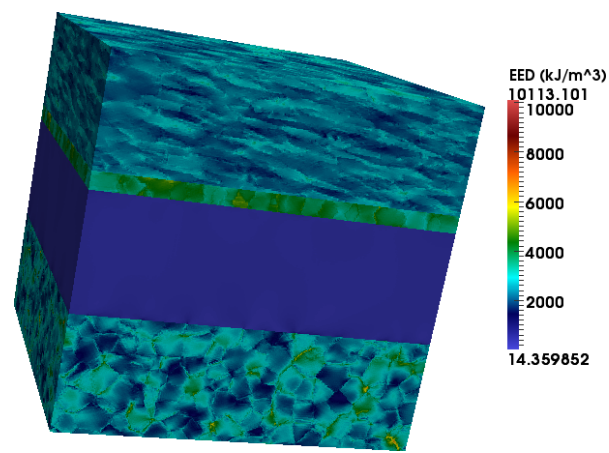

(a) NiCoCrAlY BC, randomly textured TGO, and flat inter- (b) faces.

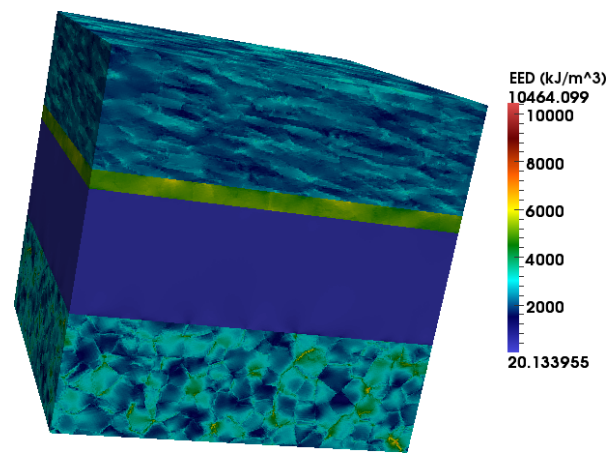

(c) NiCoCrAlY BC, fiber textured TGO, and flat interfaces.

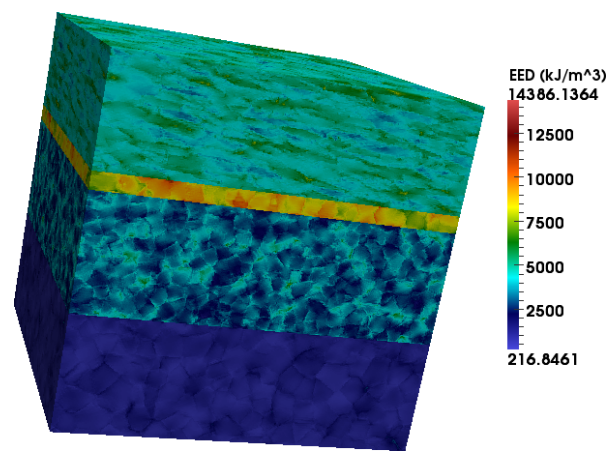

(e) $(\mathrm{Ni}, \mathrm{PT}) \mathrm{Al} \mathrm{BC}$, randomly textured TGO, and flat interfaces.

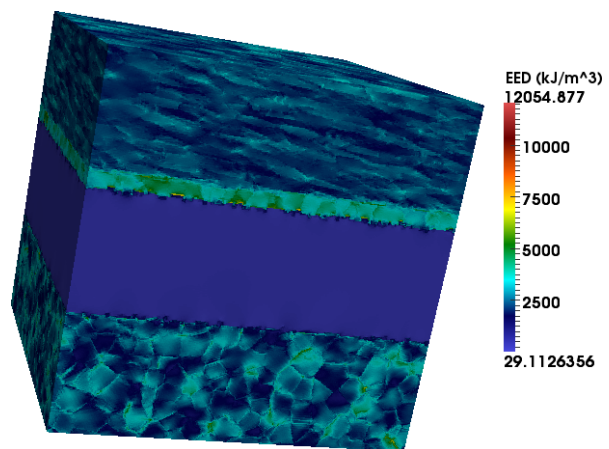

NiCoCrAlY BC, randomly textured TGO, and rumpled interfaces.

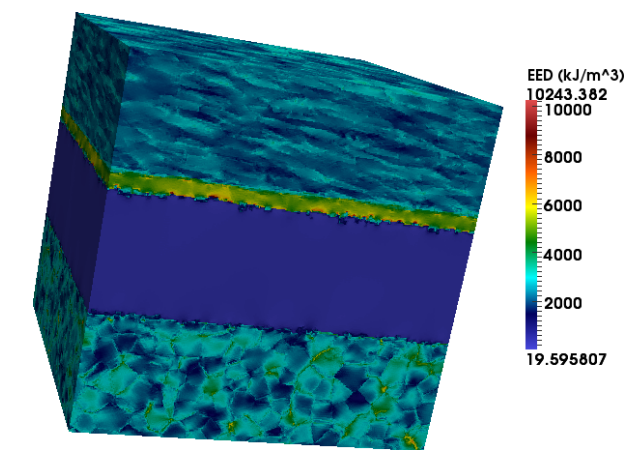

(d) NiCoCrAlY BC, fiber textured TGO, and rumpled interfaces.

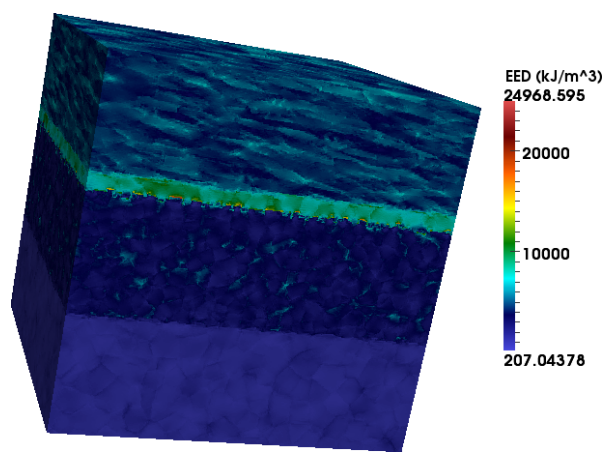

(f) (Ni,PT)Al BC, randomly textured TGO, and rumpled interfaces. 


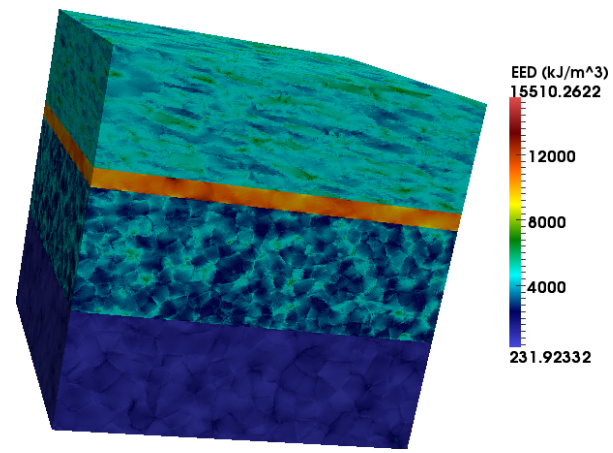

(g) $(\mathrm{Ni}, \mathrm{PT}) \mathrm{Al} \mathrm{BC}$, fiber textured TGO, and flat interfaces.

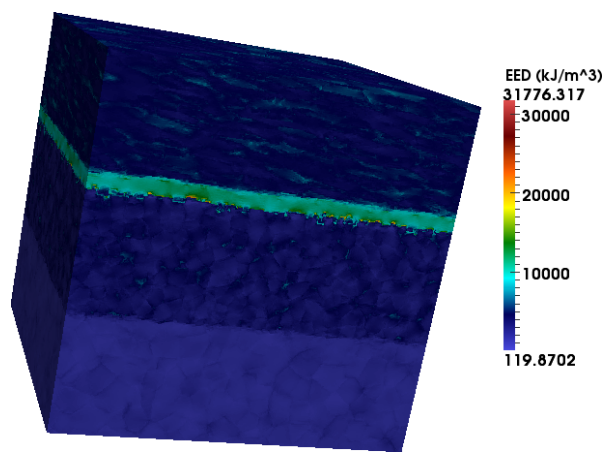

(h) $(\mathrm{Ni}, \mathrm{PT}) \mathrm{Al} \mathrm{BC}$, fiber textured TGO, and rumpled interfaces.

Figure 9: EED maps of the eight high resolution microstructures possessing splat TCs.

Again, the color maps in Figure 9 are not drawn to the same scale to highlight individual features of each microstructure. Low levels of stored strain energy in the bulk of the $\mathrm{BC}$ is again observed for the NiCoCrAlY BCs.

The TGO layer exhibits the highest level of stored strain energy, agreeing with the previously observed patterns. Peaks in EED again appear interface imperfections for the microstructures with rumpled interfaces. Table 6 lists the maximum EED for each of the simulations shown in Figure 9.

\begin{tabular}{l|c|c|c} 
Interface Type & BC Material & TGO Texture & Maximum EED $\left(\frac{\mathrm{kJ}}{\mathrm{m}^{3}}\right)$ \\
\hline \hline \multirow{4}{*}{ flat } & NiCoCrAlY & random & 10113 \\
& NiCoCrAlY & fiber & 10464 \\
& (Ni,Pt)Al & random & 14386 \\
& (Ni,Pt)Al & fiber & 15510 \\
\hline \multirow{5}{*}{ rumpled } & NiCoCrAly & random & 12055 \\
& NiCoCrAlY & fiber & 10243 \\
& (Ni,Pt)Al & random & 24969 \\
& (Ni,Pt)Al & fiber & 31776
\end{tabular}

Table 6: Highest EED for each of the simulations shown in Figure 9.

The values listed in Table 6 indicate that EED in the splat TC microstructures follows the same trends for all other microstructure with regards to interface morphology, TGO texture, and BC material. Peak EED values are generally lower than those associated with columnar TBCs. This behavior agrees with the similar observation for maximum principal stress.

\subsection{Local Variations in Elastic Energy Density}

The above results indicate a potential connection between extreme values in field variables ("hot spots") and interface rumpling. To quantify the distribution of hot spots in relation to the interface rumples, the local EED values were binned for the voxel layers near the material interfaces. Since the localized Potts model was applied to \pm 5 voxels from the initial interface (termed $z_{\text {ref }}$ ), the local EED values are binned by the same distances. The distributions within each of these bins were then drawn as boxplots. A boxplot graphically represents the range of data for a given distribution. The height of the box is the interquartile range (IQR), which is the difference between the $25^{\text {th }}$ and $75^{\text {th }}$ percentiles of the distribution. The horizontal center line is the median of the distribution. The whiskers extend above and below the box to $\frac{3}{2}$ of the IQR. Any values that lie outside these whiskers are termed outliers, and plotted discretely as points. The boxplots for the BC/TGO interfaces corresponding to randomly textured TGOs are shown in Figure 10. The color gradient corresponds to the level of elevation, with green representing low relative elevation (i.e., troughs) and red representing high relative elevation (i.e., ridges). In all cases shown, the interface troughs exhibit the 
highest level of EED. Additionally, for the NiCoCrAlY BCs, the EED values tend to decrease continuously as elevation increases. However, for the (Ni,Pt)Al BCs, the EED levels off at zero relative elevation (which corresponds to $z_{\text {ref }}$ ). Outliers appear frequently across multiple elevations. These outliers may correspond to points that sit directly next to high value points belonging to a region of low elevation. Spikes in stress values at interfaces are known to occur in FFT-based simulations, and have been tied to surface roughness at interfaces between materials [22]. Gibbs oscillations are another source of outliers in stress values and these can be mitigated by adopting a discrete Fourier transform with the necessary modifications to the Green's function [35]. In the present microstructure instantiations, the issue of surface roughness is unavoidable due to the application of the Potts model to roughen the interfaces. The outliers are therefore most likely to be artifacts of the rough interfaces.

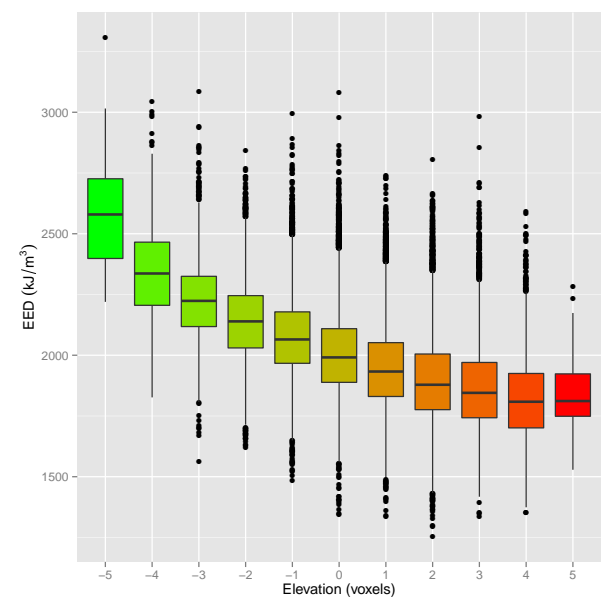

(a) columnar TC and NiCoCrAlY BC.

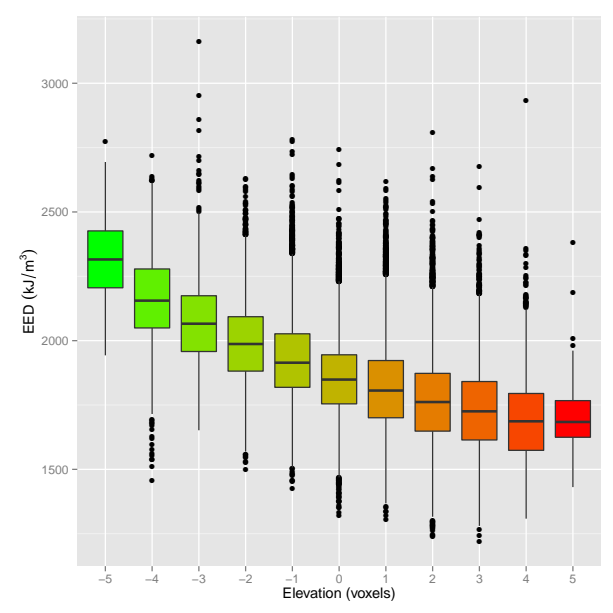

(c) splat TC and NiCoCrAlY BC.

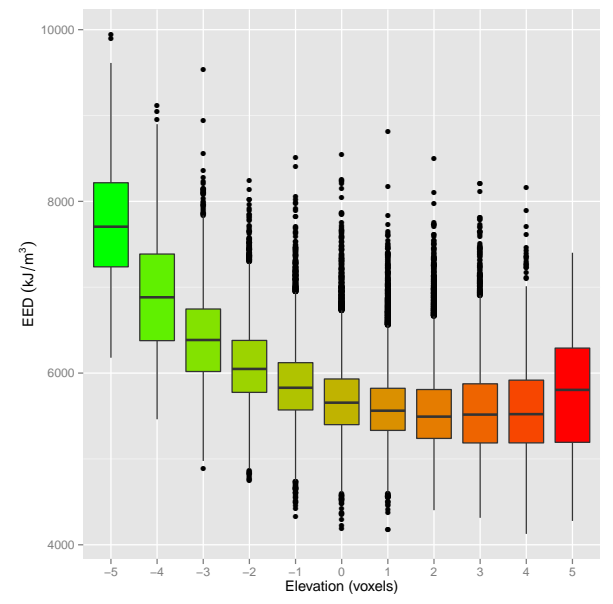

(b) columnar TC and (Ni,Pt)Al BC.

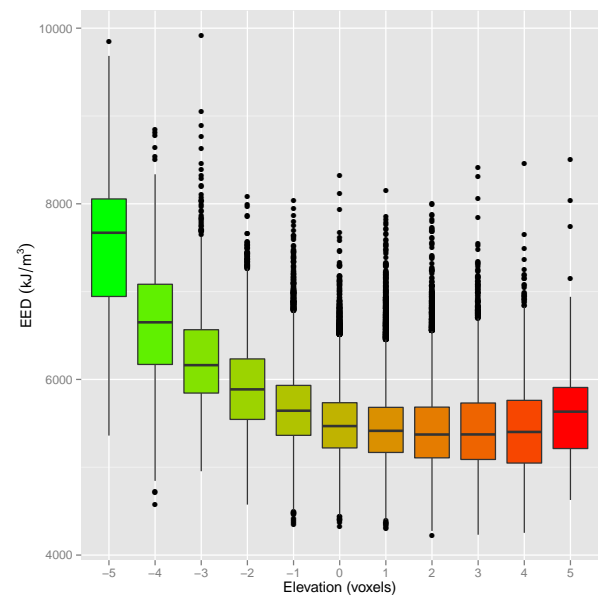

(d) splat TC and (Ni,Pt)Al BC.

Figure 10: Boxplots for the EED distributions of the BC/TGO interfaces corresponding to randomly textured TGOs, binned by elevation.

The same procedure used to draw the boxplots shown in Figure 10 can be applied to the BC/TGO interfaces with a fiber textured TGO. These boxplots are shown in Figure 11. The same general trends are observed for the case of a fiber textured TGO. EED exhibits a peak within the troughs and decreases with increasing elevation. 


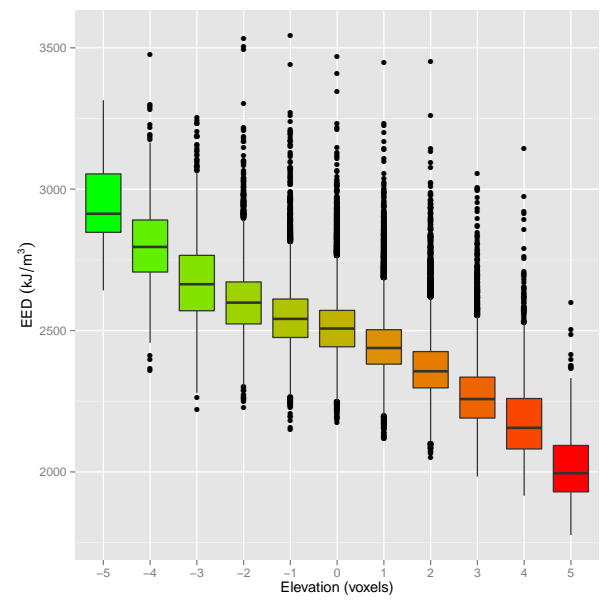

(a) columnar TC and NiCoCrAlY BC.

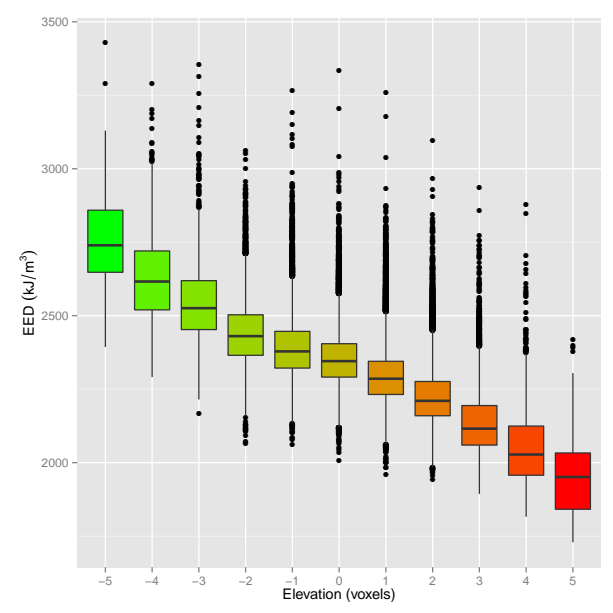

(c) splat TC and NiCoCrAlY BC.

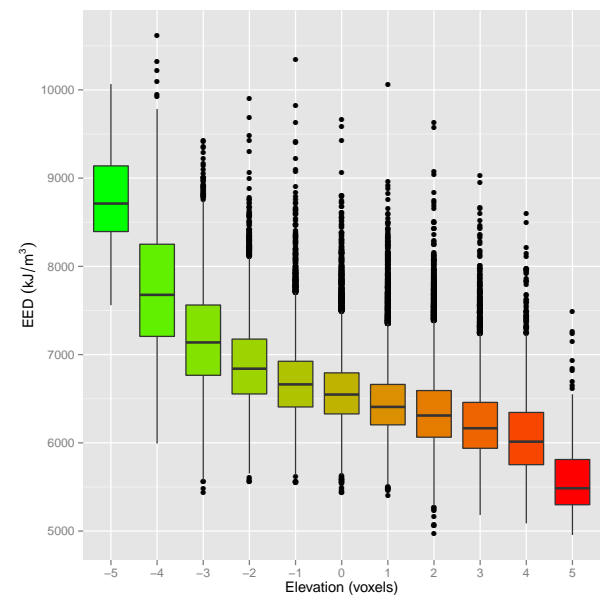

(b) columnar TC and (Ni,Pt)Al BC.

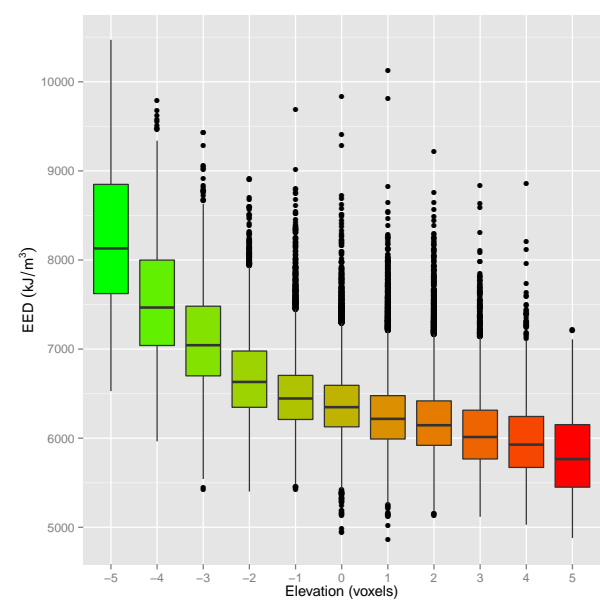

(d) splat TC and (Ni,Pt)Al BC.

Figure 11: Boxplots for the EED distributions of the BC/TGO interfaces corresponding to fiber textured TGOs, binned by elevation.

Similar to the interfaces with randomly textured TGOs, a number of outliers are observed; again, these may be points that directly border points of low elevation. The trends in Figure 11 agree with those in Figure 10, indicating that the texture of the TGO does not impact the overall trend of EED with respect to interface rumpling at the $\mathrm{BC} / \mathrm{TGO}$ interface.

Compared to the $\mathrm{BC} / \mathrm{TGO}$ interfaces, the TGO/TC interfaces exhibit completely different behavior. Figure 12 shows the boxplots for the EED distributions of the TGO/TC interfaces with random TGO texture. 


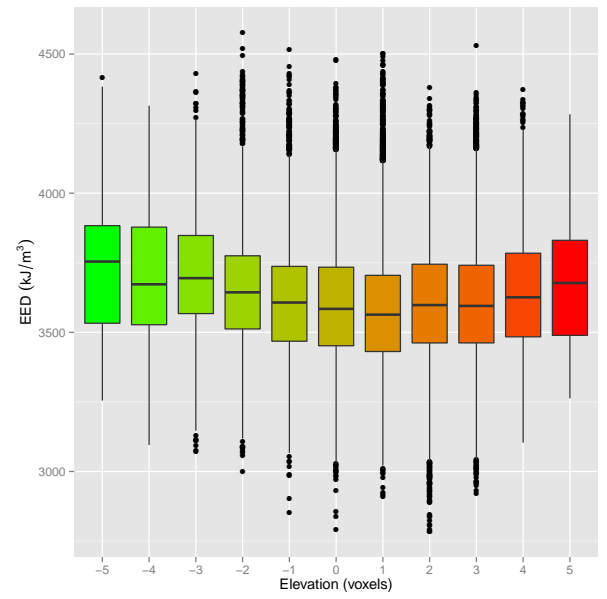

(a) columnar TC and NiCoCrAlY BC.

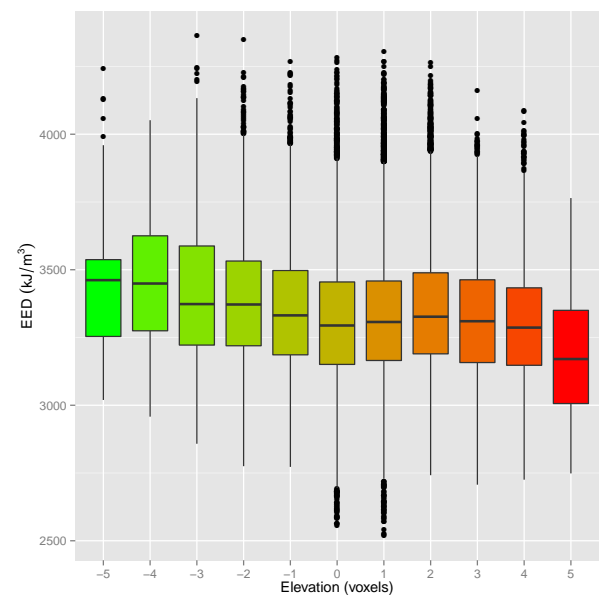

(c) splat TC and NiCoCrAlY BC.

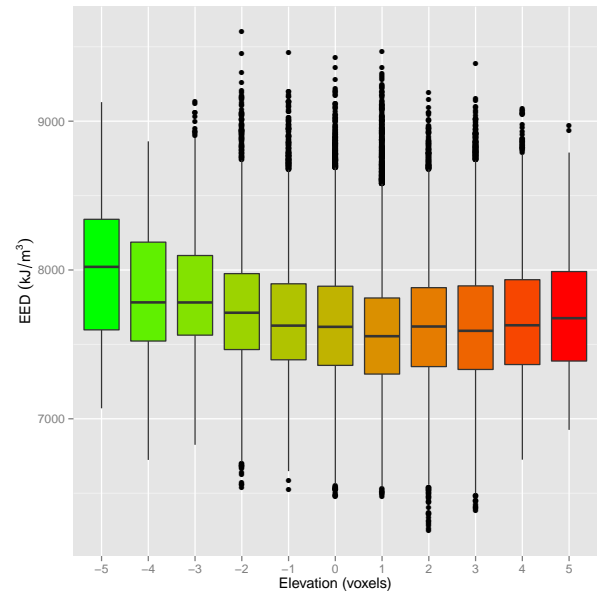

(b) columnar TC and (Ni,Pt)Al BC.

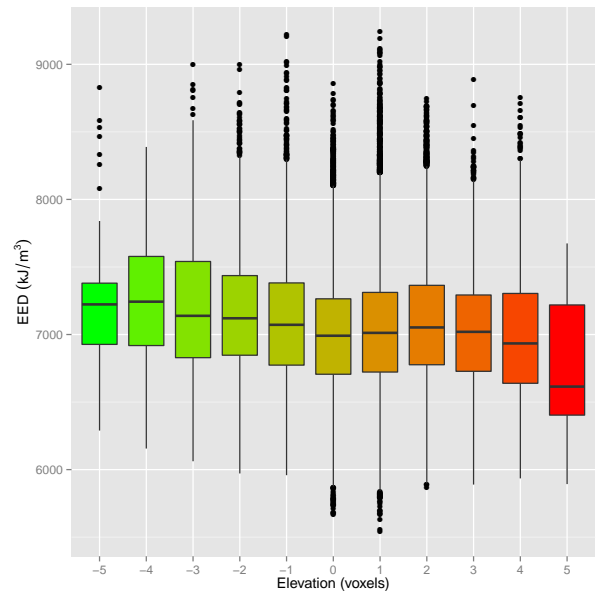

(d) splat TC and (Ni,Pt)Al BC.

Figure 12: Boxplots for the EED distributions of the TGO/TC interfaces corresponding to randomly textured TGOs, binned by elevation.

The boxplots shown in Figure 12 show no correlation between elevation and amount of EED. Therefore, unlike for the case at the $\mathrm{BC} / \mathrm{TGO}$ interface, the amount of rumpling does not appear to influence the distribution of EED across the surface. The same lack of correlation is observed for the TGO/TC interfaces with a fiber textured TGO, as shown in Figure 13. 


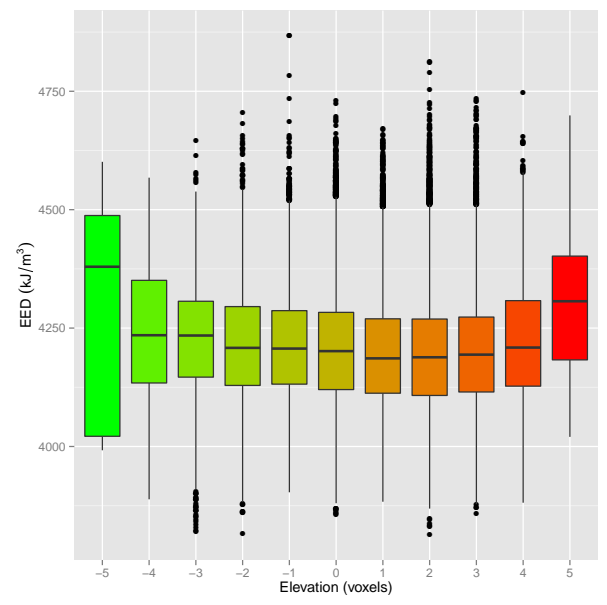

(a) columnar TC and NiCoCrAlY BC.

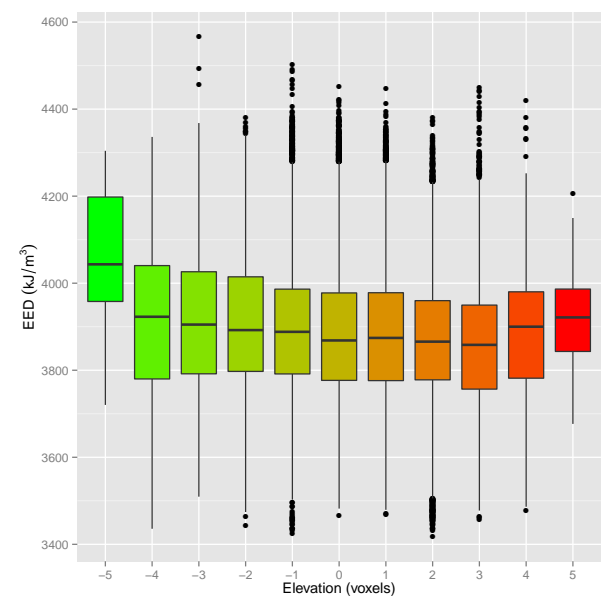

(c) splat TC and NiCoCrAlY BC.

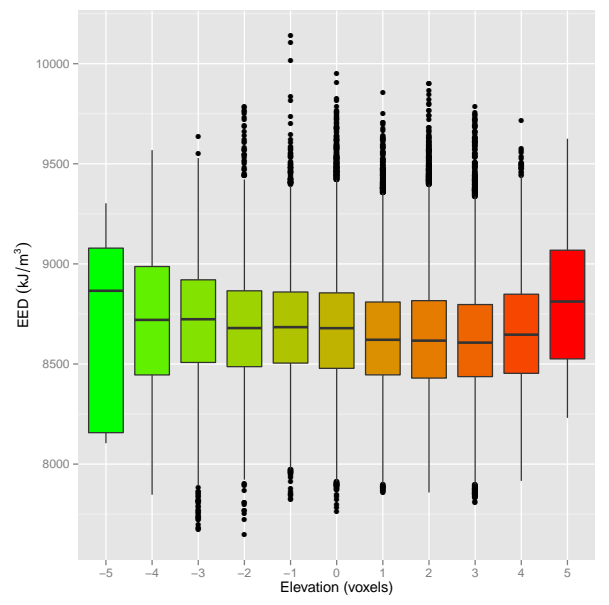

(b) columnar TC and (Ni,Pt)Al BC.

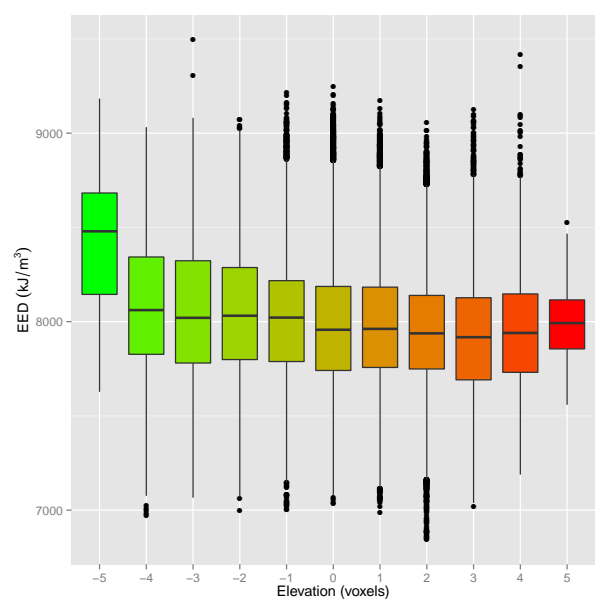

(d) splat TC and (Ni,Pt)Al BC.

Figure 13: Boxplots for the EED distributions of the TGO/TC interfaces corresponding to fiber textured TGOs, binned by elevation.

Again, the amount of EED does not appear to have a direct correlation with elevation at the TGO/TC interface. For columnar TCs, there may be a spike in EED at interface ridges, however the IQR at these features is large enough to overlap with the IQR at other elevations. Therefore, unlike for the BC/TGO interfaces, the relation between hot spots and rumpling at the TGO/TC interfaces appears weak.

\section{Discussion}

Comparisons between the behavior of the $\mathrm{BC} / \mathrm{TGO}$ and TGO/TC interfaces reveal a correlation between EED and interface roughness at the BC/TGO interface but not at the TGO/TC interface. The amount of stored strain energy, however, is comparable between the two interface types, which is apparent by comparing the scales of Figures 11 and 13. However, the fluctuations in stress within the plane of the BC/TGO interface may serve as a driving force for crack nucleation. Initial crack formation in TBCs often occurs at the $\mathrm{BC} /$ TGO interface, which may ultimately serve as the failure plane of the system $[6,9,15,16]$. The present results concerning the simulated EED gradient at the BC/TGO interface offer an possible explanation for the local driving force for failure.

During thermal expansion, two bonded layers of differing thermomechanical properties must experience tensile and compressive strains to remain continuously bonded [10]. In the presence of a rumpled or wavy 
interface, substantial stresses normal to the interface may be exhibited. It is possible to derive an analytical expression for the upper bound for the behavior of the normal stresses along a roughened interface between two layers, assuming the interface undulations are sinusoidal and the morphology of the coating is cylindrical [10]:

$$
\sigma_{n} \approx \Delta \epsilon \frac{\left(\frac{1}{\kappa}+E_{c}\left(1+\nu_{s}\right)\left(\frac{R}{a}\right)\right) \sin \left(\frac{2 \pi y}{\lambda}\right)}{1+\frac{R}{t}}
$$

where $\sigma_{n}$ is the stress normal to the interface at position $y, R$ is the amplitude of the undulations, $\lambda$ is the undulations, $t$ is the thickness of the coating layer, and $\Delta \epsilon$ is the mismatch strain, defined as:

$$
\Delta \epsilon=\Delta \alpha \Delta T
$$

where $\Delta \alpha$ is the difference in thermal expansion between the two layers and $\Delta T$ is the applied temperature difference. Equation 23 also involves the Young's modulus of the top layer, $E_{c}$, and the Poisson's ratio of the substrate layer, $\nu_{s}$. The value $\kappa$ is a further relation between the mechanical properties of the two layers, and is defined as follows:

$$
\kappa=\frac{1+\nu_{c}}{2 E_{c}}+\frac{1-2 \nu_{s}}{E_{s}}
$$

where $\nu_{c}$ and $E_{s}$ are the Poisson's ratio and Young's modulus of the top layer and substrate layer, respectively. Equation 23 can be used to approximate the normal stresses along a sinusoidal interface between two bonded layers of differing thermomechanical properties. To compare to the present simulation results, equation 23 was evaluated for the material properties of the BC/TGO and TGO/TC interfaces for a (Ni,Pt)Al BC. Table 7 lists the material properties used to approximate equation 23.

\begin{tabular}{l|c|c|c} 
Material & $E(\mathrm{GPa})$ & $\nu$ & $\alpha\left(10^{-6} \mathrm{~K}^{-1}\right)$ \\
\hline \hline$(\mathrm{Ni}, \mathrm{Pt}) \mathrm{Al}$ & 110 & 0.3 & 15.5 \\
\hline$\alpha-\mathrm{Al}_{2} \mathrm{O}_{3}$ & 400 & 0.25 & 8.095 \\
\hline $\mathrm{YSZ}$ & 100 & 0.12 & 11
\end{tabular}

Table 7: Material properties for analytical evaluation of normal stresses [9, 36, 37].

The values listed in Table 8 were used to approximate the geometric features of the interface undulations.

\begin{tabular}{l|c|c|c|c} 
Interface Type $\mid \mathrm{R}(\mu \mathrm{m})$ & $\mathrm{a}(\mu \mathrm{m})$ & $\lambda(\mu \mathrm{m})$ & $\mathrm{t}(\mu \mathrm{m})$ \\
\hline \hline $\mathrm{BC} / \mathrm{TGO}$ & 10 & 10 & 0.5 & 15 \\
\hline $\mathrm{TGO} / \mathrm{TC}$ & 10 & 10 & 0.5 & 100
\end{tabular}

Table 8: Interface properties for analytical evaluation of normal stresses.

Since the synthetic structures were instantiated with a per voxel resolution of about $1 \mu \mathrm{m}$, an amplitude of 10 $\mu \mathrm{m}$ was used to correspond to the maximum change in elevation of \pm 5 voxels. Similarly, the thicknesses were set to 15 and $100 \mu \mathrm{m}$, to correspond to the voxel thicknesses of the TGO and TC, respectively. The radius and wavelength values are difficult to assign proper values for the present investigation, since the synthetic interfaces do not conform to a cylindrical geometry and the interface undulations are far from sinusoidal. Approximate values of 10 and $0.5 \mu \mathrm{m}$ were used for these quantities. To complete the comparison with the simulation results, a $\Delta \mathrm{T}$ of $1000 \mathrm{~K}$ was used. The results of equation 23 when evaluated with the parameters in Tables 7 and 8 are shown in Figure 14. 


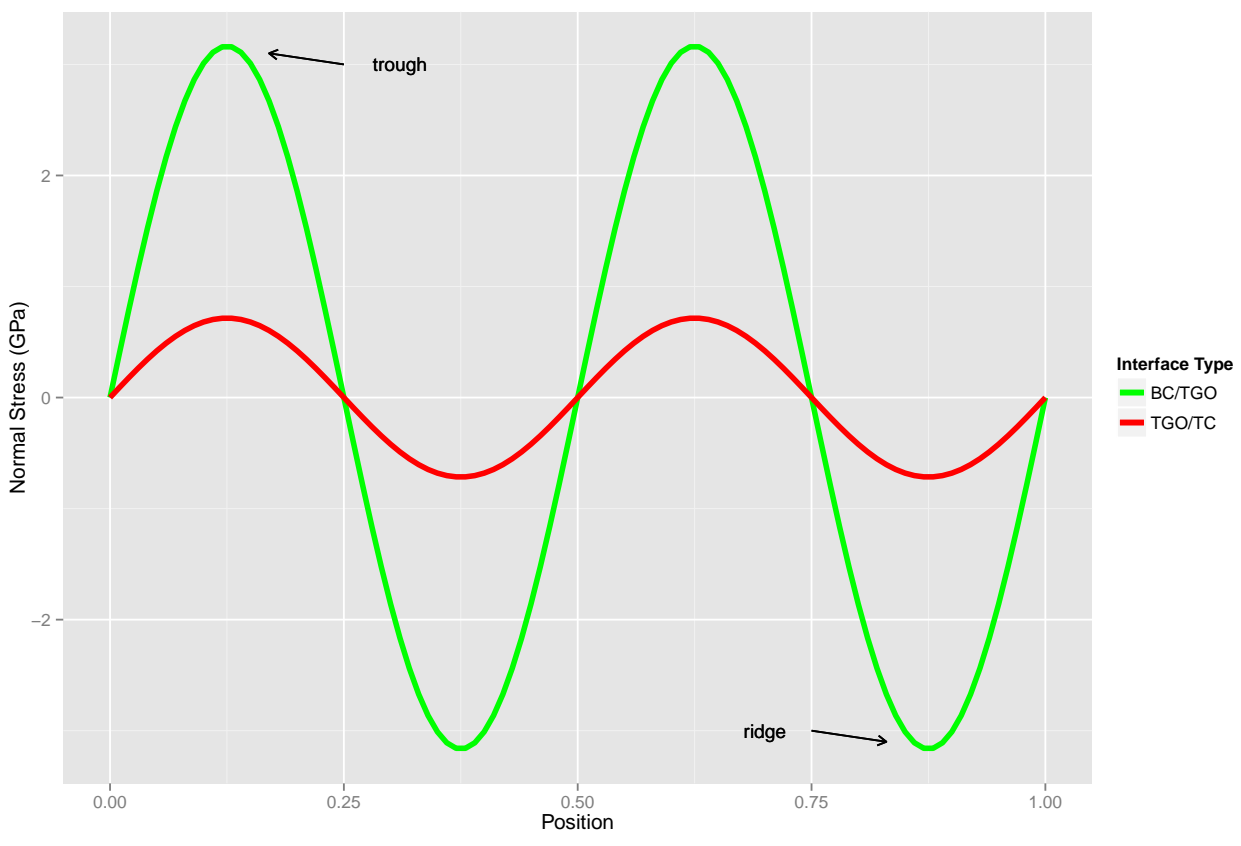

Figure 14: Analytical results for normal stresses along undulating interfaces.

The position of the troughs and ridges of the sinusoidal interface are labeled in Figure 14. The normal stresses vary with position similarly between the $\mathrm{BC} / \mathrm{TGO}$ and $\mathrm{TGO} / \mathrm{TC}$ interfaces, but the peak values are much larger for the BC/TGO interface, likely due to the higher mismatch strain between the BC and TGO compared to the TGO and TC. The stress values are tensile at the troughs and compressive at the ridges. To compare to the simulation results, the normal stresses with computed for the two interface types for the case of the EB-PVD TC and (Ni,Pt)Al BC with fiber textured TGO and plotted against elevation using the boxplot approach. Figure 15 shows the boxplots for the two interface types.

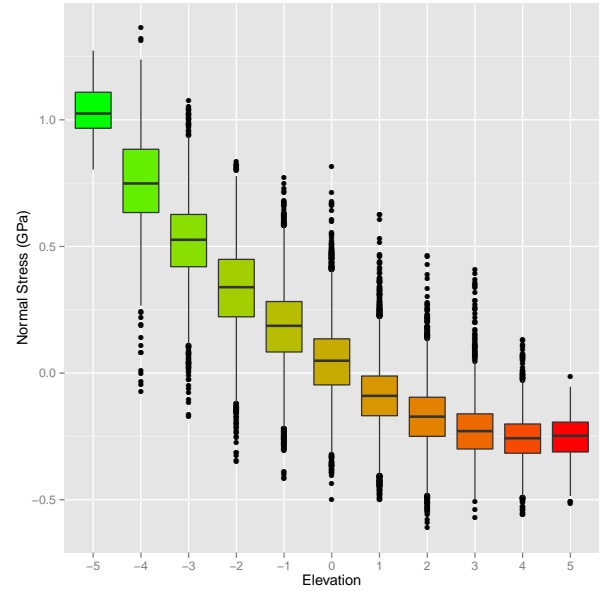

(a) BC/TGO interface.

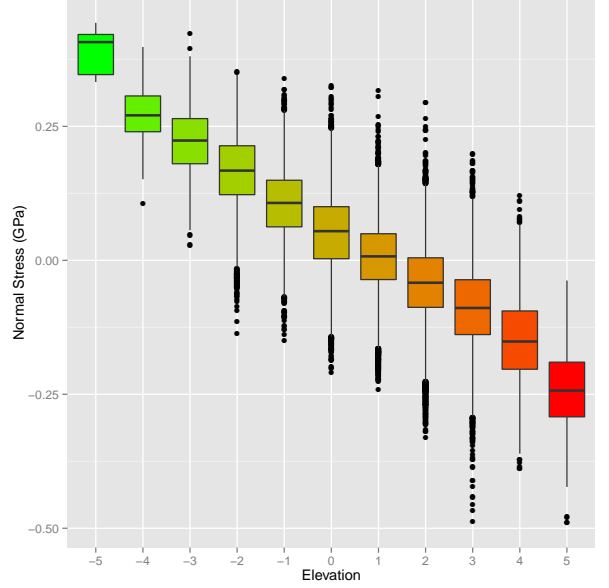

(b) TGO/TC interface.

Figure 15: Boxplots for the normal stress distributions of EB-PVD TC and (Ni,Pt)Al BC system with fiber textured TGOs, binned by elevation.

Figure 15 shows that despite the morphological differences between the analytical geometry and the synthetic microstructures, the overall trend between normal stress and elevation agree: tensile stresses are associated with low elevations (troughs) and compressive stresses are associated with high elevations (ridges). Addi- 
tionally, the magnitude of the normal stresses from the simulation results roughly agree with the analytical results, although the simulation tends to underestimate compared to the analytical results. This discrepancy may be due to the stochastic nature of the synthetic interface, as opposed to a continuous sinusoidal interface.

\section{Conclusions}

A simulation technique for computing residual stresses and strains based on FFTs was applied to high resolution synthetic microstructures intended to represent TBC systems. Several various microstructure instantiations were created with various microstructure morphologies, such columnar structures from EBPVD processes or splat structures from APS application. The texture in the TGO layer was also varied to be either random or fiber textured. To approximate rumpling at the TBC interfaces, a localized Potts model was applied to the voxel layers near the interfaces. The results indicate that interface rumpling increases both the maximum stress and maximum EED in the microstructures. Additionally, microstructures with fiber textured TGOs exhibited higher residual stresses than those with randomly textured TGOs. The EED values near the interfaces were also related with the level of interface rumpling. A correlation between EED and interface elevation was observed at the BC/TGO interface, but not at the TGO/TC interface. Additionally, the simulation results compare favorably to analytical results for ideal sinusoidal interfaces.

The present results indicate that the thermoelastic FFT method is a powerful technique for analyzing the thermomechanical behavior of complex systems such as TBCs. A strong advantage of the technique is its ability to asses local microstructural properties while maintaining reasonable scaling. Although the microstructures used here were generated synthetically, the technique is capable of utilizing images obtained experimentally as direct inputs. However, the thermoelastic approach does not include any plastic deformation, which may be present in TBC systems in the form of interface ratcheting and creep. The technique also does not allow for crack nucleation or growth. These limitations will need to be addressed so that future versions of the technique will be able to assess TBC behavior beyond the local origins of failure.

\section{Acknowledgements}

This work was primarily supported by grant number DE-FE0003840 from the Department of Energy as part of the University Coal Research Program. The authors are grateful to Drs. Ben Anglin and Ricardo Lebensohn for extensive discussions and development of the FFT simulation code.

\section{References}

[1] X. Zhang, B. Xu, H. Wang, Y. Jiang, , Y. Wu, Modeling of thermal residual stresses in multilayer coatings with graded properties and compositions, Thin Solid Films 497 (2006) 223-231.

[2] R. Miller, Current status of thermal barrier coatings - an overview, Surf. Coat. Tech. 30 (1987) 1-11.

[3] D. Clarke, C. Levi, Materials design for the next generation thermal barrier coatings, Annu. Rev. Materi. Res. 33 (2003) 383-417.

[4] D. Clarke, S. Phillpot, Thermal barrier coating materials, Mater. Today 8 (2005) 22-29.

[5] R. Vaßen, M. Jarligo, T. Steinke, D. Mack, D. Stöver, Overview on advanced thermal barrier coating materials, Surf. Coat. Tech. 205 (2010) 938-942.

[6] N. Padture, M. Gell, E. Jordan, Thermal barrier coatings for gas-turbine engine applications, Science 296 (2002) 280-284.

[7] J. Cheng, E. Jordan, B. Barber, M. Gell, Thermal/residual stress in an electron beam physical vapor deposited thermal barrier coating system, Acta Mater. 46 (1998) 5839-5850.

[8] W. Brindley, Properties of plasma-sprayed bond coats, J. Therm. Spray Tech. 6 (1997) 85-90. 
[9] A. Evans, D. M. amd J.W. Hutchinson, G. Meier, F. Pettit, Mechanisms controlling the durability of thermal barrier coatings, Prog. Mater. Sci. 46 (2001) 505-553.

[10] A. Evans, G. Crumley, R. Demaray, On the mechanical behavior of brittle coatings and layers, Oxid. Met. 20 (1983) 193-216.

[11] X. Gong, D. Clarke, On the measurement of strain in coatings formed on a wrinkled elastic substrate, Oxid. Met. 50 (1998) 355-376.

[12] M. He, A. Evans, J. Hutchinson, The ratcheting of compressed thermally grown thin films on ductile substrates, Acta Mater. 48 (2000) 2593-2601.

[13] E. Shillington, D. Clarke, Spalling failure of a thermal barrier coating associated with aluminum depletion in the bond-coat, Acta Mater. 47 (1999) 1297-1305.

[14] W. Brindley, R. Miller, Thermal barrier coating life and isothermal oxidation of low-pressure plasmasprayed bond coat alloys, Surf. Coat. Tech. 43 (1990) 446-457.

[15] K. Schlichting, N. Padture, E. Jordan, M. Gell, Failure modes in plasma-sprayed thermal barrier coatings, Mater. Sci. Eng. A 342 (2003) 120-130.

[16] R. Miller, C. Lowell, Failure mechanisms of thermal barrier coatings exposed to elevated temperatures, Metall. Prot. Coat. (1982) 265-273.

[17] P. Bengtsson, C. Persson, Modelled and measured residual stress in plasma sprayed thermal barrier coatings, Surf. Coat. Tech. 92 (1997) 78-86.

[18] H. Li, L. Sun, Z. Wang, X-ray stress measurement and FEM analysis of residual stress distribution near interface in bonded ceramic/metal compounds, Scripta Mater. 34 (1996) 1503-1508.

[19] R. Lebensohn, N-site modeling of a 3D viscoplastic polycrystal using fast fourier transform, Acta Mater. 49 (2001) 2723-2737.

[20] J. Eshelby, The determination of the elastic field of an ellipsoidal inclusion and related problems, P. Roy. Soc. Lond. A Mat. 241 (1957) 376-396.

[21] J. Eshelby, The elastic fields outside an ellipsoidal inclusion, P. Roy. Soc. Lond. A Mat. 252 (1959) $561-569$.

[22] B. Anglin, R. Lebensohn, A. Rollett, Validation of a numerical method based on fast fourier transforms for heterogeneous thermoelastic materials by comparison with analytical solutions, Comp. Materi. Sci. 87 (2014) 209-217.

[23] E. Schumann, C. Sarioglu, J. Blachere, F. Pettit, G. Meier, High-temperature stress measurements during the oxidation of NiAl, Oxid. Met. 53 (2000) 259-272.

[24] D. Lipkin, D. Clarke, Measurement of the stress in oxide scales formed by oxidation of alumina-forming alloys, Oxid. Met. 45 (1996) 267-280.

[25] K. Schlichting, K. Vaidyanathan, Y. Sohn, E. Jordan, M. Gell, N. Padture, Application of Cr ${ }^{3+}$ photoluminescence piezo-spectroscopy to plasma-sprayed thermal barrier coatings for residual stress measurement, Mater. Sci. Eng. A 291 (2000) 68-77.

[26] S. Berbenni, M. Cherkaoui, Homogenization of multi-coated inclusion-reinforced linear elastic composites with eigenstrains: Application to the thermo-elastic behavior, Phil. Mag. 90 (2010) 3003-3026.

[27] B. Anglin, Ph.D. thesis, Carnegie Mellon University, Pittsburgh, PA (2012).

[28] H. Moulinec, P. Suquet, A numerical method for computing the overall response of nonlinear composites with complex microstructure, Comput. Meth. Appl. Mech. Eng. 157 (1998) 69-94. 
[29] T. Mura, Micromechanics of Defects in Solids, Kluwer Academic Publishers (1991).

[30] J. Michel, H. Moulinec, P. Suquet, A computational scheme for linear and non-linear composites with arbitrary phase contrast, Comput. Meth. Appl. Mech. Eng. 1 (2001) 79-88.

[31] J. Michel, H. Moulinec, P. Suquet, A computational method based on augmented lagrangians and fast fourier transforms for composites with high contrast, Int. J. Numer. Meth. Eng. 52 (2000) 139-160.

[32] M. Groeber, M. Jackson, DREAM.3D: A digital representation environment for the analysis of microstructure in 3D, Integr. Mater. Manuf. Innov. 3 (2014).

[33] F. Wu, The Potts model, Rev. Mod. Phys. 54 (1982) 235-268.

[34] R. Lebensohn, R. Brenner, O. Castelnau, A. Rollett, Orientation image-based micromechanical modelling of subgrain texture evolution in polycrystalline copper, Acta Mater. 56 (2008) 3914-3926.

[35] S. Berbenni, V. Taupin, K. Djaka, C. Fressengeas, A numerical spectral approach for solving elasto-static field dislocation and g-disclination mechanics, Int. J. Solids Struct. 51 (2014) 4157-4175.

[36] E. Brusso, H. Evans, Z. Qian, M. Taylor, Effects of breakaway oxidation on local stresses in thermal barrier coatings, Acta Mater. 58 (2010) 1242-1251.

[37] M. Watanabe, D. Mumm, S. Chiras, A. Evans, Measurement of the residual stress in a Pt aluminide bond coat, Scripta Mater. 46 (2002) 67-70. 


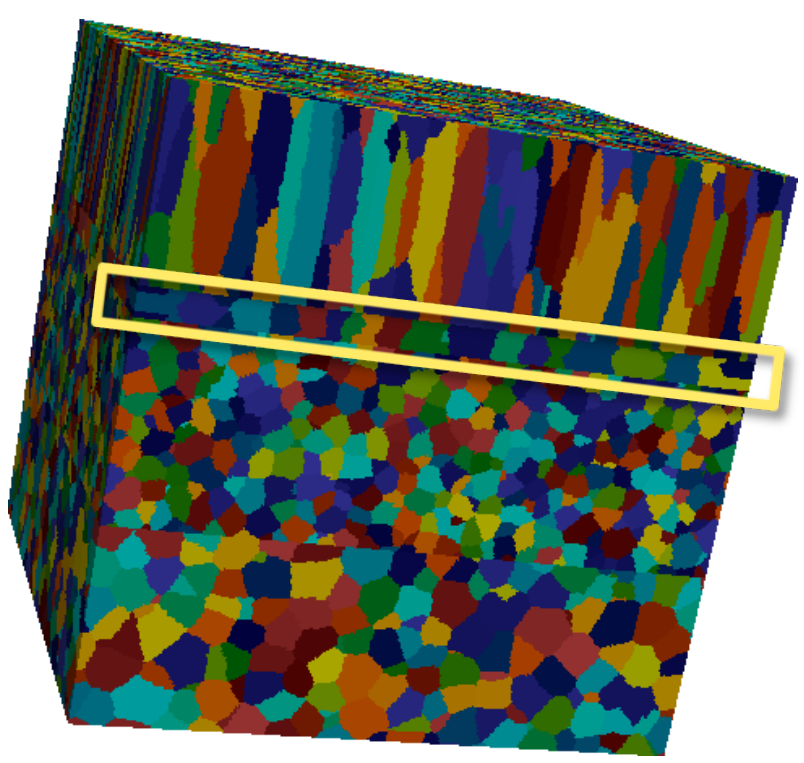

High Resolution Synthetic Microstructure
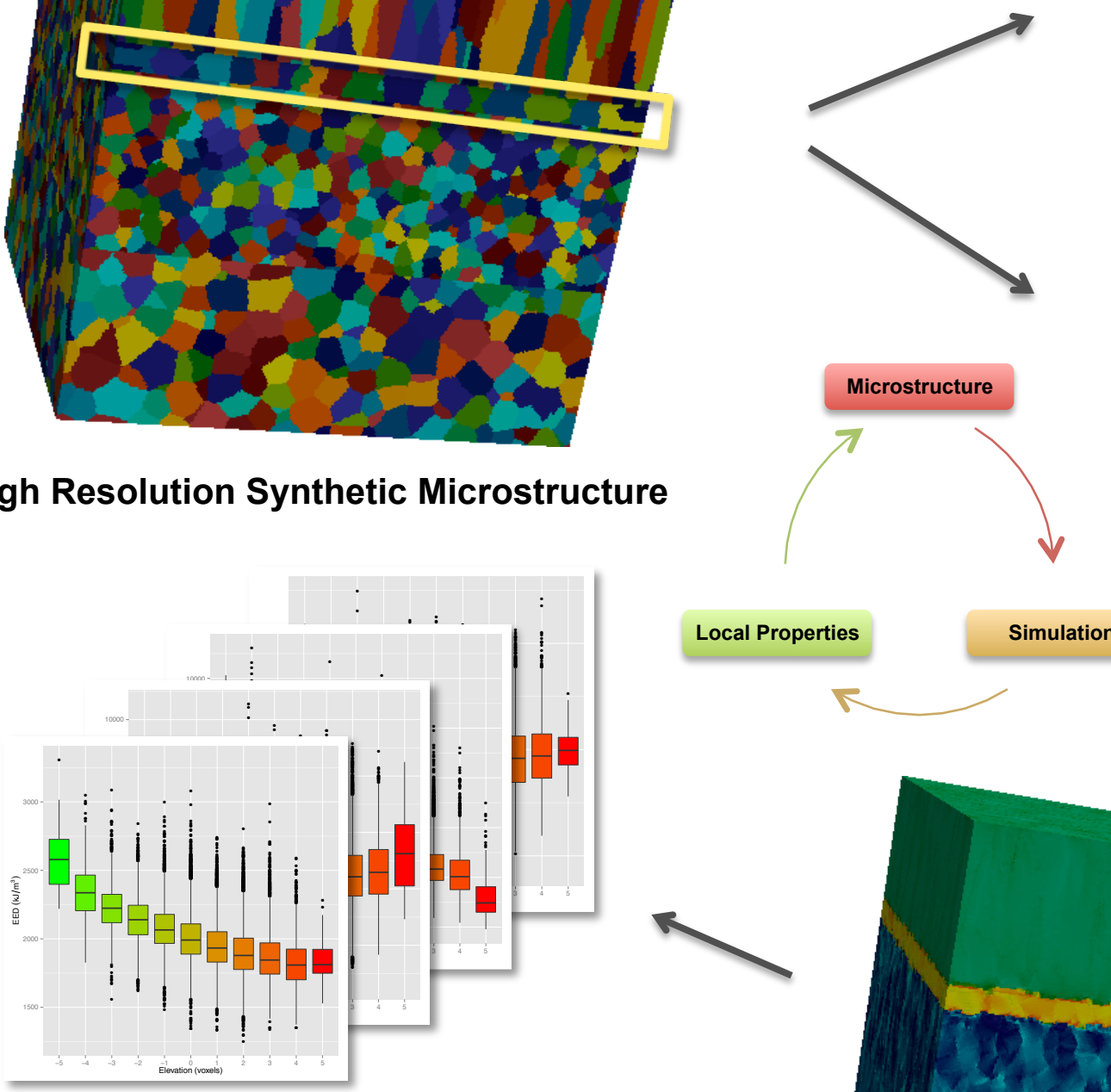

Stochastic Interface Variations

Statistical Analysis

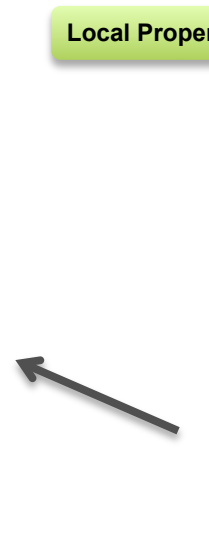

R
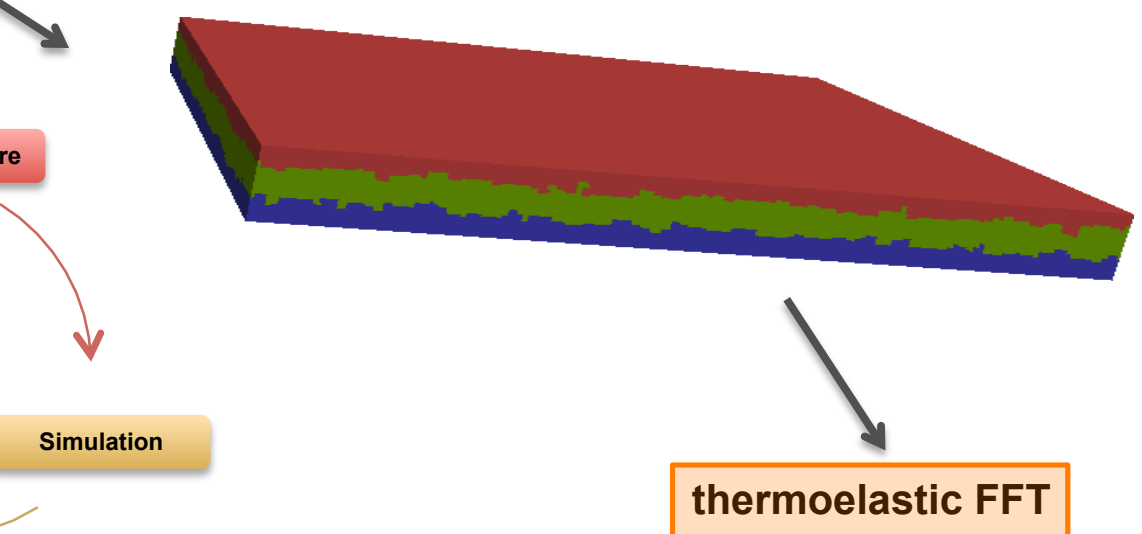

thermoelastic FFT
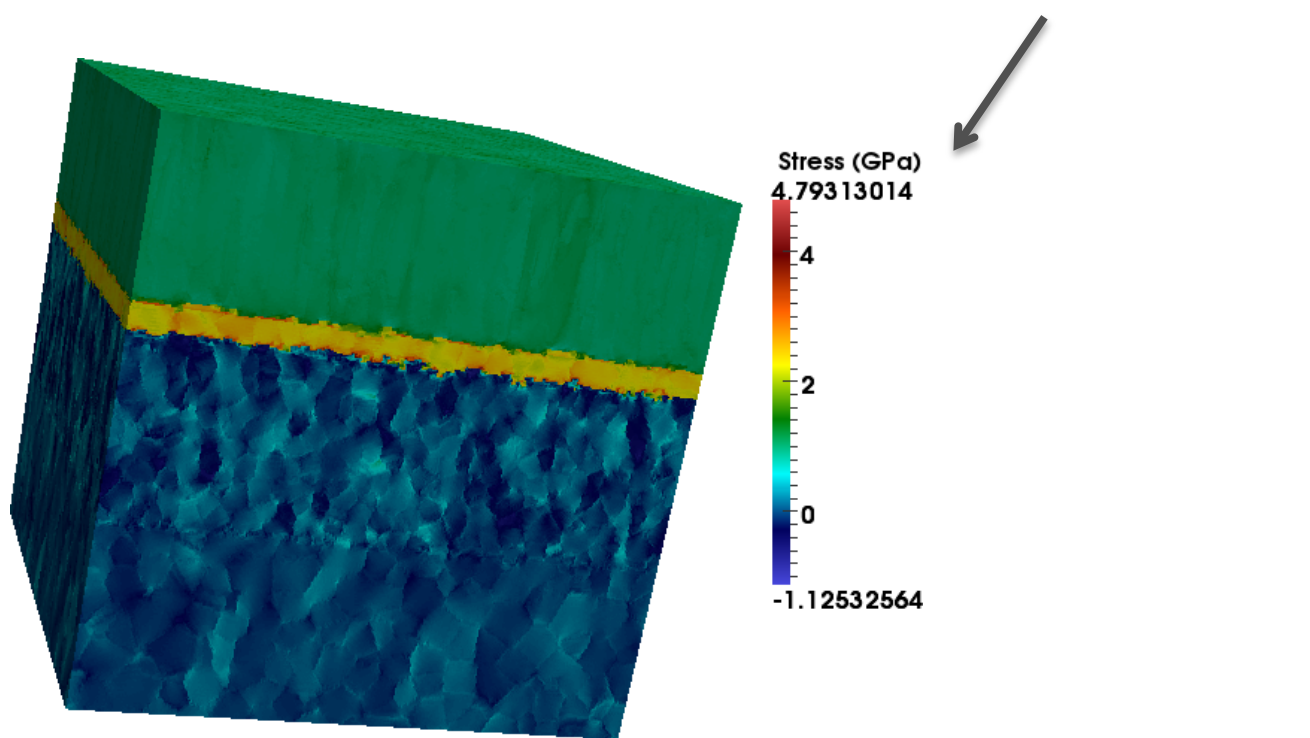